ESAIM: M2AN

Vol. 40, No 6, 2006, pp. 1023-1052

DOI: $10.1051 / \mathrm{m} 2 \mathrm{an}: 2006039$
ESAIM: Mathematical Modelling and Numerical Analysis

www.edpsciences.org/m2an

\title{
PERIODIC SOLUTIONS FOR NONLINEAR ELLIPTIC EQUATIONS. APPLICATION TO CHARGED PARTICLE BEAM FOCUSING SYSTEMS
}

\author{
Mihai Bostan ${ }^{1}$ And ERIC SONNEndRÜCKER ${ }^{2}$
}

\begin{abstract}
We study the existence of spatial periodic solutions for nonlinear elliptic equations $-\Delta u+g(x, u(x))=0, x \in \mathbb{R}^{N}$ where $g$ is a continuous function, nondecreasing w.r.t. $u$. We give necessary and sufficient conditions for the existence of periodic solutions. Some cases with nonincreasing functions $g$ are investigated as well. As an application we analyze the mathematical model of electron beam focusing system and we prove the existence of positive periodic solutions for the envelope equation. We present also numerical simulations.
\end{abstract}

Mathematics Subject Classification. 35A05, 35B35.

Received: April 10, 2006.

\section{INTRODUCTION}

The main model used for studying beam propagation is the Vlasov equation coupled with the Maxwell or Poisson equations. It describes the evolution of populations of charged particles under the effects of external and self-consistent electro-magnetic fields. Since the numerical simulation of solutions for the Vlasov-Maxwell system requires important computational efforts, it is worth to take into account the particularities of the physical problem (typical lengths, geometric and physical characteristics) to derive approximate simplified models. One of the models which is often used in Accelerator Physics for analyzing propagation of beams possessing an optical axis is the Paraxial model. For a physicist's derivation of this model one can refer to the book by Davidson and Qin [4]. A rigorous study of the paraxial model was done by Degond and Raviart [5, 13]. They give a complete analysis of the linear model and present the KV (Kapchinsky-Vladimirsky) distributions, see also [7], which are exact solutions of the paraxial model. The case of high energy short beams is studied by Laval $e t$ al. in [9] and the case of axisymmetric laminar beams is analyzed by Nouri in [12]. Techniques for focusing fairly general particle beams rely on the focusing of KV beams and the concept of equivalent beams [4]. Thus, if a focused KV beam can be found for a given accelerating system, a general beam with the same moments up to order two will be approximately focused. Moreover, a way to find a focused KV beam is to find periodic solutions of the so-called envelope equation (see $[4,6,11,14]$ )

$$
-u^{\prime \prime}(x)-a k(x) u(x)+\frac{1}{u(x)}+\frac{b}{(u(x))^{3}}=0, \quad x \in \mathbb{R},
$$

Keywords and phrases. Nonlinear elliptic equations, periodic solutions, existence and uniqueness, electron beam focusing system.

1 Laboratoire de Mathématiques de Besançon, UMR CNRS 6623, Université de Franche-Comté, 16 route de Gray, 25030 Besançon Cedex, France. mbostan@math.univ-fcomte.fr

2 IRMA, Université Louis Pasteur, 7 rue René Descartes, 67084 Strasbourg Cedex, France. sonnen@math.u-strasbg.fr 
where $a>0, b \geq 0$ are some constants and $k(\cdot)$ is a given nonnegative periodic function corresponding to the periodic magnetic device. We are looking for periodic solutions $u$ with the same period as $k(\cdot)$. More generally we consider nonlinear elliptic equations of type

$$
-\Delta u+g(x, u(x))=0, \quad x \in \mathbb{R}^{N},
$$

where $g: \mathbb{R}^{N} \times \mathbb{R} \rightarrow \mathbb{R}$ is a given function. Two sorts of nonlinearities $g$ will be considered : nondecreasing and nonincreasing. The best situation is when the nonlinearity is nondecreasing. In this case we assume that

$\left(H_{1}\right) \quad g(x, \cdot)$ is continuous and nondecreasing a.e. $x \in \mathbb{R}^{N}$;

$\left(H_{2}\right) \quad g$ is periodic w.r.t. $x$, i.e., $\exists L=\left(L_{1}, L_{2}, \ldots, L_{N}\right) \in\left(\mathbb{R}_{+}^{\star}\right)^{N}$ such that $g\left(x_{1}+k_{1} L_{1}, \ldots, x_{N}+k_{N} L_{N}, u\right)=g\left(x_{1}, \ldots, x_{N}, u\right)$, a.e. $x \in \mathbb{R}^{N}, \forall u \in \mathbb{R}$, $\forall\left(k_{1}, \ldots, k_{N}\right) \in \mathbb{Z}^{N} ;$

$\left(H_{3}\right) \quad \forall R>0, \exists C_{R}:\left|\nabla_{x}\{g(x, u)-g(x, 0)\}\right| \leq C_{R}$, a.e. $x \in \mathbb{R}^{N},|u| \leq R$ if $N=1$, $\left|\nabla_{x}\{g(x, u)-g(x, 0)\}\right| \leq C_{g}|u|^{p}$, a.e. $x \in \mathbb{R}^{N}, u \in \mathbb{R}$, for some $1 \leq p<\infty$ if $N=2$ and $1 \leq p \leq \frac{N+2}{N-2}$ if $N \geq 3$

$\left(H_{4}\right) \quad g(\cdot, 0) \in L_{\mathrm{loc}}^{2}\left(\mathbb{R}^{N}\right)$.

Consider $P=\left\{x \in \mathbb{R}^{N}: 0 \leq x_{1}<L_{1}, 0 \leq x_{2}<L_{2}, \ldots, 0 \leq x_{N}<L_{N}\right\}$ and for any $k \in \mathbb{N}$ denote by $C_{\#}^{k}\left(\mathbb{R}^{N}\right)$ the space of $\left(L_{1}, L_{2}, \ldots, L_{N}\right)$ periodic functions of $C^{k}\left(\mathbb{R}^{N}\right)$. We introduce also the periodic Sobolev space

$$
\begin{aligned}
H_{\#}^{1}\left(\mathbb{R}^{N}\right)=\left\{v \in L_{\mathrm{loc}}^{2}\left(\mathbb{R}^{N}\right):\right. & \exists\left(\varphi_{n}\right)_{n} \subset C_{\#}^{1}\left(\mathbb{R}^{N}\right), \lim _{n \rightarrow+\infty}\left\|v-\varphi_{n}\right\|_{L_{\mathrm{loc}}^{2}\left(\mathbb{R}^{N}\right)=0} \\
& \left.\lim _{n, m \rightarrow+\infty}\left\|\nabla \varphi_{n}-\nabla \varphi_{m}\right\|_{L_{\mathrm{loc}}^{2}\left(\mathbb{R}^{N}\right)}=0\right\} .
\end{aligned}
$$

Observe that for any $v \in H_{\#}^{1}\left(\mathbb{R}^{N}\right)$ we can associate $\nabla v=\lim _{n \rightarrow+\infty} \nabla \varphi_{n}$ in $L_{\text {loc }}^{2}\left(\mathbb{R}^{N}\right)$ which depends only on $v$ and not on the sequence $\left(\varphi_{n}\right)_{n}$. We consider the inner product

$$
\langle u, v\rangle_{H_{\#}^{1}\left(\mathbb{R}^{N}\right)}=\int_{P} u(x) v(x) \mathrm{d} x+\int_{P} \nabla u \cdot \nabla v \mathrm{~d} x, \quad \forall u, v \in H_{\#}^{1}\left(\mathbb{R}^{N}\right),
$$

and we obtain the Hilbert space $\left(H_{\#}^{1}\left(\mathbb{R}^{N}\right),\langle\cdot, \cdot\rangle_{H_{\#}^{1}\left(\mathbb{R}^{N}\right)}\right)$. Observe also that we have the following formula of integration by parts

$$
\int_{P} u(x) \frac{\partial v}{\partial x_{i}} \mathrm{~d} x+\int_{P} \frac{\partial u}{\partial x_{i}} v(x) \mathrm{d} x=0, \forall u, v \in H_{\#}^{1}\left(\mathbb{R}^{N}\right), 1 \leq i \leq N
$$

Actually we have the equivalent definition

$$
H_{\#}^{1}\left(\mathbb{R}^{N}\right)=\left\{v \in H_{\mathrm{loc}}^{1}\left(\mathbb{R}^{N}\right): \int_{P+K L}\{\nabla v \varphi(x)+v(x) \nabla \varphi\} \mathrm{d} x=0, \forall \varphi \in C_{\#}^{1}\left(\mathbb{R}^{N}\right), \forall K \in \mathbb{Z}^{N}\right\},
$$


where $P+K L=\left\{\left(x_{1}+k_{1} L_{1}, x_{2}+k_{2} L_{2}, \ldots, x_{N}+k_{N} L_{N}\right), \quad\left(x_{1}, x_{2}, \ldots, x_{N}\right) \in P\right\}$ for any $K=\left(k_{1}, k_{2}, \ldots, k_{N}\right) \in \mathbb{Z}^{N}$. From the last definition we deduce that $H_{\#}^{1}\left(\mathbb{R}^{N}\right)$ is closed in $H_{\text {loc }}^{1}\left(\mathbb{R}^{N}\right)$. We introduce also

$$
\begin{aligned}
H_{\#}^{2}\left(\mathbb{R}^{N}\right)= & \left\{v \in L_{\mathrm{loc}}^{2}\left(\mathbb{R}^{N}\right): \exists\left(\varphi_{n}\right)_{n} \subset C_{\#}^{2}\left(\mathbb{R}^{N}\right), \lim _{n \rightarrow+\infty}\left\|v-\varphi_{n}\right\|_{L_{\mathrm{loc}}^{2}\left(\mathbb{R}^{N}\right)}=0,\right. \\
& \left.\lim _{n, m \rightarrow+\infty}\left\|\nabla \varphi_{n}-\nabla \varphi_{m}\right\|_{L_{\mathrm{loc}}^{2}\left(\mathbb{R}^{N}\right)}=0, \lim _{n, m \rightarrow+\infty}\left\|D^{2} \varphi_{n}-D^{2} \varphi_{m}\right\|_{L_{\mathrm{loc}}^{2}\left(\mathbb{R}^{N}\right)}=0\right\},
\end{aligned}
$$

where $D^{2} \varphi:=\left(\partial_{x_{i} x_{j}}^{2} \varphi\right)_{1 \leq i, j \leq N}$ for any function $\varphi \in C^{2}\left(\mathbb{R}^{N}\right)$. We will use the notation $\|u\|_{L_{\#}^{q}\left(\mathbb{R}^{N}\right)}=$ $\left(\int_{P}|u(x)|^{q} \mathrm{~d} x\right)^{1 / q}$ for any $L$ periodic function in $L_{\mathrm{loc}}^{q}\left(\mathbb{R}^{N}\right), 1 \leq q<+\infty$.

Definition 1.1. We say that $u \in H_{\#}^{1}\left(\mathbb{R}^{N}\right)$ is a $L=\left(L_{1}, L_{2}, \ldots, L_{N}\right)$ periodic solution for $(2)$ iff $x \rightarrow g(x, u(x))$ belongs to $L_{\text {loc }}^{2}\left(\mathbb{R}^{N}\right)$ and

$$
\int_{P} \nabla u \cdot \nabla v \mathrm{~d} x+\int_{P} g(x, u(x)) v(x) \mathrm{d} x=0, \quad \forall v \in H_{\#}^{1}\left(\mathbb{R}^{N}\right) .
$$

Notice that the function appearing in (1) is nonincreasing w.r.t. $u$. Actually we will see that in some cases existence results are available also for nonincreasing functions $g$. One of the key points of our analysis is to observe that the existence of periodic solution for (2) requires additional necessary conditions on the function $g$. For example, in one dimension, assume that there is a periodic (smooth) solution for

$$
-u^{\prime \prime}(x)+g(x, u(x))=0, \quad x \in \mathbb{R},
$$

with $g$ continuous, $L$ periodic (nondecreasing or nonincreasing). Denote by $G: \mathbb{R} \rightarrow \mathbb{R}$ the function

$$
G(u)=\int_{0}^{L} g(x, u) \mathrm{d} x, \quad u \in \mathbb{R},
$$

which is also a monotone continuous function. After integration of (4) w.r.t. $x$ over one period one gets

$$
\int_{0}^{L} g(x, u(x)) \mathrm{d} x=0 .
$$

If $u$ is bounded we can write $m \leq u(x) \leq M, x \in \mathbb{R}$ and by monotonicity we obtain $G(m) G(M) \leq 0$. Finally one gets that $G$ vanishes at some point $u_{0} \in \mathbb{R}$ and therefore a necessary condition for the existence of periodic solution is $0 \in \operatorname{Range}(G)$. Conversely, when $g$ is nondecreasing w.r.t. $u$, we prove that the condition $0 \in \operatorname{Int}(\operatorname{Range}(G))$ guarantees the existence of periodic solution. We have the main result

Theorem 1.1. Assume that $g: \mathbb{R}^{N} \times \mathbb{R} \rightarrow \mathbb{R}$ satisfies $\left(H_{1}\right),\left(H_{2}\right),\left(H_{3}\right),\left(H_{4}\right)$ and $0 \in \operatorname{Int}\left(\operatorname{Range}\left(\int_{P} g(x, \cdot) \mathrm{d} x\right)\right)$. Then there is at least one periodic solution $u \in H_{\#}^{2}\left(\mathbb{R}^{N}\right)$ for $(2)$. If $g$ is strictly increasing w.r.t. $u$ then the periodic solution is unique.

In the particular case $g(x, u)=\beta(u)-f(x),(x, u) \in \mathbb{R}^{N} \times \mathbb{R}, 1 \leq N \leq 3$ we obtain

Theorem 1.2. Assume that $\beta: \mathbb{R} \rightarrow \mathbb{R}$ is continuous, nondecreasing, $\beta(0)=0, f \in L_{\#}^{2}\left(\mathbb{R}^{N}\right), 1 \leq N \leq 3$ and that $\langle f\rangle:=(\operatorname{meas}(P))^{-1} \int_{P} f(x) \mathrm{d} x \in \operatorname{Range}(\beta)$. Then there is at least one periodic solution $u \in H_{\#}^{2}\left(\mathbb{R}^{N}\right)$ for

$$
-\Delta u+\beta(u(x))=f(x), \quad x \in \mathbb{R}^{N},
$$

satisfying

$$
\left|\langle u\rangle-\tilde{u}_{0}\right|+\left\|u-\tilde{u}_{0}\right\|_{H_{\#}^{2}\left(\mathbb{R}^{N}\right)} \leq C\|f-\langle f\rangle\|_{L_{\#}^{2}\left(\mathbb{R}^{N}\right)},\|\beta(u)-\langle f\rangle\|_{L_{\#}^{2}\left(\mathbb{R}^{N}\right)} \leq\|f-\langle f\rangle\|_{L_{\#}^{2}\left(\mathbb{R}^{N}\right)},
$$


where $\tilde{u}_{0}$ is the element of minimal absolute value of the closed convex set $\beta^{-1}\langle f\rangle \neq \emptyset$. If $\beta$ is strictly increasing then the periodic solution is unique.

In one dimension we analyze also the existence of periodic solution for

$$
-u^{\prime \prime}(x)-g(x, u(x))=0, \quad x \in \mathbb{R},
$$

where $g$ is nondecreasing w.r.t. $u$.

Similar results were obtained for first order differential equations $u^{\prime}(t)+g(t, u(t))=0, t \in \mathbb{R}$ and also for evolution equations $\frac{\mathrm{d} u}{\mathrm{~d} t}+A u(t)=f(t), t \in \mathbb{R}$, where $A: D(A) \subset H \rightarrow H$ is a linear, symmetric, maximal monotone operator on a Hilbert space and $f$ is a $T$ periodic function. In this last case we prove that there is a $T$ periodic solution iff $\langle f\rangle:=\frac{1}{T} \int_{0}^{T} f(t) \mathrm{d} t \in \operatorname{Range}(A)$. For more details the reader can refer to $[1,2]$.

The paper is organized as follows. In Section 2 we analyze the case of nondecreasing nonlinearities. We construct periodic solutions for penalized problems. After establishing uniform estimates one gets the existence of periodic solution by passing the penalization parameter towards 0 . The solution constructed by the above procedure satisfies a minimality property and is uniquely determined by this property. We present a stability result for the minimal periodic solution. We study also the asymptotic behavior of the minimal periodic solution for large frequencies. In Section 3 we investigate the case of nonincreasing nonlinearities in one dimension. We obtain similar results provided that the nonlinearity is $K$ Lipschitz w.r.t. $u$ with $K$ small enough. We end this paper with several numerical simulations. We compute approximations for the periodic solutions of the envelope equation in one dimension.

\section{EXISTENCE OF PERIODIC SOLUTION FOR NONDECREASING NONLINEARITIES}

In this section we suppose that $g$ is nondecreasing w.r.t. $u$. Throughout this study we will introduce several necessary conditions on the function $g$ for the existence of periodic solution.

\subsection{Necessary conditions for existence of periodic solution}

By taking $v=1$ in (3) we deduce that $\int_{P} g(x, u(x)) \mathrm{d} x=0$, meaning that a necessary condition for the existence of periodic solution for (2) is

$$
\left(C_{1}\right) \quad \exists u \in H_{\#}^{1}\left(\mathbb{R}^{N}\right): g(\cdot, u(\cdot)) \in L_{\mathrm{loc}}^{1}\left(\mathbb{R}^{N}\right), \int_{P} g(x, u(x)) \mathrm{d} x=0 .
$$

We assume also that

$$
\left(H_{5}\right) \quad g(\cdot, u) \in L_{\mathrm{loc}}^{1}\left(\mathbb{R}^{N}\right), \quad \forall u \in \mathbb{R},
$$

(observe that this happens under the hypothesis $\left(H_{3}\right)$ ) and we introduce the function $G(u)=\int_{P} g(x, u) \mathrm{d} x$, $u \in \mathbb{R}$. Under the hypothesis $\left(H_{1}\right)$ we check easily that $G(\cdot)$ is nondecreasing and continuous. Another hypothesis appearing through our analysis will be

$$
\left(C_{2}\right) \quad \exists u_{0} \in \mathbb{R}: G\left(u_{0}\right)=\int_{P} g\left(x, u_{0}\right) \mathrm{d} x=0 .
$$

Obviously $\left(C_{2}\right)$ is stronger than $\left(C_{1}\right)$ but if the function $u(\cdot)$ in $\left(C_{1}\right)$ is bounded we can prove as in the introduction that $\left(C_{1}\right)$ and $\left(C_{2}\right)$ are equivalent. The functions of $H_{\#}^{1}(\mathbb{R})$ are continuous and bounded. Hence we have

Proposition 2.1. Assume that $g$ satisfies $\left(H_{1}\right),\left(H_{5}\right)$ and that $\left(C_{1}\right)$ holds with a bounded function $u \in L^{\infty}\left(\mathbb{R}^{N}\right)$. Then $\left(C_{2}\right)$ holds too. In particular, if $N=1$, the conditions $\left(C_{1}\right)$ and $\left(C_{2}\right)$ are equivalent. 
Generally $\left(C_{1}\right)$ does not imply $\left(C_{2}\right)$. Nevertheless $\left(C_{1}\right)$ implies the following condition

$$
\left(C_{3}\right) \quad 0 \in \overline{\text { Range }(G)} \text {. }
$$

Proposition 2.2. Assume that $g$ satisfies $\left(H_{1}\right),\left(H_{5}\right)$ and that $\left(C_{1}\right)$ holds. Then $\left(C_{3}\right)$ holds too.

Proof. For $n \geq 1$ consider $g_{n}(x)=g(x, \min \{n, u(x)\}), x \in \mathbb{R}^{N}$. Observe that we have for any $n \geq 1$

$$
\min \{g(x, 0), g(x, u(x))\}=g(x, \min \{0, u(x)\}) \leq g_{n}(x) \leq g(x, u(x)), \text { a.e. } x \in \mathbb{R}^{N} \text {. }
$$

The sequence $\left(g_{n}(x)\right)_{n}$ is nondecreasing and converges towards $g(x, u(x))$ a.e. $x \in \mathbb{R}^{N}$. By using the Lebesgue dominated convergence theorem we deduce that $\lim _{n \rightarrow+\infty} g_{n}=g(\cdot, u(\cdot))$ in $L_{\text {loc }}^{1}\left(\mathbb{R}^{N}\right)$. Therefore we have

$$
0=\int_{P} g(x, u(x)) \mathrm{d} x=\lim _{n \rightarrow+\infty} \int_{P} g_{n}(x) \mathrm{d} x \leq \lim _{n \rightarrow+\infty} \int_{P} g(x, n) \mathrm{d} x=\lim _{n \rightarrow+\infty} G(n) .
$$

Take now $\tilde{g}_{n}=g(x, \max \{-n, u(x)\})$. Observe that we have for any $n \geq 1$

$$
g(x, u(x)) \leq \tilde{g}_{n}(x) \leq g(x, \max \{0, u(x)\})=\max \{g(x, 0), g(x, u(x))\}, \text { a.e. } x \in \mathbb{R}^{N} .
$$

The sequence $\left(\tilde{g}_{n}(x)\right)_{n}$ is nonincreasing, converges towards $g(x, u(x))$ a.e. $x \in \mathbb{R}^{N}$ and therefore $\lim _{n \rightarrow+\infty} \tilde{g}_{n}=$ $g(\cdot, u(\cdot))$ in $L_{\text {loc }}^{1}\left(\mathbb{R}^{N}\right)$. As before we have

$$
0=\int_{P} g(x, u(x)) \mathrm{d} x=\lim _{n \rightarrow+\infty} \int_{P} \tilde{g}_{n}(x) \mathrm{d} x \geq \lim _{n \rightarrow+\infty} \int_{P} g(x,-n) \mathrm{d} x=\lim _{n \rightarrow+\infty} G(-n) .
$$

We proved that $\lim _{v \rightarrow-\infty} G(v) \leq 0 \leq \lim _{v \rightarrow+\infty} G(v)$ and therefore $0 \in \overline{\operatorname{Range}(G)}$.

Remark 2.1. The Propositions 2.1 and 2.2 hold also true for functions $g$ nonincreasing w.r.t. $u$.

As we will see later on, the existence of periodic solution is established under the condition

$\left(C_{4}\right) \quad 0 \in \operatorname{Int}($ Range $(G))$

which is stronger than the necessary condition $\left(C_{1}\right)$ (actually we have the implications $\left(C_{4}\right) \Longrightarrow\left(C_{2}\right) \Longrightarrow$ $\left.\left(C_{1}\right) \Longrightarrow\left(C_{3}\right)\right)$. We investigate now a class of functions $g$ for which conditions $\left(C_{4}\right)$ and $\left(C_{1}\right)$ coincide and thus become a necessary and sufficient condition for the existence of periodic solution for (2).

Definition 2.1. Assume that $g: \mathbb{R}^{N} \times \mathbb{R} \rightarrow \mathbb{R}$ is a function satisfying $\left(H_{1}\right),\left(C_{1}\right)$.

1) We say that $g$ is strictly increasing at $+\infty$ if there is a measurable set $A^{+} \subset P, \operatorname{meas}\left(A^{+}\right)>0$ such that

$$
g(x, u(x))<\lim _{v \rightarrow+\infty} g(x, v), \text { a.e. } x \in A^{+}
$$

2) We say that $g$ is strictly decreasing at $-\infty$ if there is a measurable set $A^{-} \subset P, \operatorname{meas}\left(A^{-}\right)>0$ such that

$$
g(x, u(x))>\lim _{v \rightarrow-\infty} g(x, v), \text { a.e. } x \in A^{-} .
$$

Notice that (7) is equivalent to

$$
\exists n_{0} \in \mathbb{N}: \int_{P \cap\left\{x: u(x) \leq n_{0}\right\}} g(x, u(x)) \mathrm{d} x<\int_{P \cap\left\{x: u(x) \leq n_{0}\right\}} g\left(x, n_{0}\right) \mathrm{d} x,
$$


and also that (8) is equivalent to

$$
\exists m_{0} \in \mathbb{N}: \int_{P \cap\left\{x: u(x) \geq-m_{0}\right\}} g(x, u(x)) \mathrm{d} x>\int_{P \cap\left\{x: u(x) \geq-m_{0}\right\}} g\left(x,-m_{0}\right) \mathrm{d} x .
$$

Proposition 2.3. Assume that $g: \mathbb{R}^{N} \times \mathbb{R} \rightarrow \mathbb{R}$ is a function satisfying $\left(H_{1}\right),\left(H_{5}\right),\left(C_{1}\right)$.

1) If $g$ is strictly increasing at $+\infty$ then there is $u_{0}^{+} \in \mathbb{R}$ such that $G\left(u_{0}^{+}\right)>0$;

2) if $g$ is strictly decreasing at $-\infty$ then there is $u_{0}^{-} \in \mathbb{R}$ such that $G\left(u_{0}^{-}\right)<0$;

3) if $g$ is strictly increasing at $+\infty$ and strictly decreasing at $-\infty$ then $\left(C_{4}\right)$ holds true.

Proof. Let us prove the statement 1 . Take $u$ satisfying $\left(C_{1}\right)$. For any $n \geq 1$ we consider $A_{n}=\{x \in P: u(x) \leq$ $n\}$. Since $u \in L_{\#}^{2}\left(\mathbb{R}^{N}\right)$ we have

$$
\int_{P}|u(x)|^{2} \mathrm{~d} x \geq \int_{P \cap \complement A_{n}}|u(x)|^{2} \mathrm{~d} x \geq \operatorname{meas}\left(P \cap \complement A_{n}\right) n^{2}
$$

and thus $\lim _{n \rightarrow+\infty} \operatorname{meas}\left(P \cap \complement A_{n}\right)=0$. We denote by $\left(a_{n}\right)_{n}$ the sequence

$$
a_{n}=\int_{A_{n}}\{g(x, n)-g(x, u(x))\} \mathrm{d} x, \quad n \geq 1 .
$$

Since $A_{n} \subset A_{n+1}, n \geq 1$ we can write for any $n \geq 1$

$$
0 \leq a_{n} \leq \int_{A_{n}}\{g(x, n+1)-g(x, u(x))\} \mathrm{d} x \leq \int_{A_{n+1}}\{g(x, n+1)-g(x, u(x))\} \mathrm{d} x=a_{n+1},
$$

and we deduce that $\left(a_{n}\right)_{n}$ is nondecreasing. As $g$ is strictly increasing at $+\infty$ we deduce that $\exists n_{0} \in \mathbb{N}: a_{n} \geq$ $a_{n_{0}}>0, n \geq n_{0}$. Observe that

$$
0 \leq \int_{P \cap \complement A_{n}}\{g(x, u(x))-g(x, n)\} \mathrm{d} x \leq \int_{P \cap \complement A_{n}}\{g(x, u(x))-g(x, 0)\} \mathrm{d} x \rightarrow 0,
$$

as $n \rightarrow+\infty$, since $g(\cdot, u(\cdot)), g(\cdot, 0)$ belong to $L_{\text {loc }}^{1}\left(\mathbb{R}^{N}\right)$ and $\lim _{n \rightarrow+\infty} \operatorname{meas}\left(P \cap \complement A_{n}\right)=0$. Take now $n_{1} \geq n_{0}$ large enough such that

Finally we deduce

$$
\int_{P \cap \complement A_{n_{1}}}\left\{g(x, u(x))-g\left(x, n_{1}\right)\right\} \mathrm{d} x<\frac{a_{n_{0}}}{2} .
$$

$$
\begin{aligned}
\int_{P}\left\{g\left(x, n_{1}\right)-g(x, u(x))\right\} \mathrm{d} x & =\int_{A_{n_{1}}}\left\{g\left(x, n_{1}\right)-g(x, u(x))\right\} \mathrm{d} x-\int_{P \cap \complement A_{n_{1}}}\left\{g(x, u(x))-g\left(x, n_{1}\right)\right\} \mathrm{d} x \\
& =a_{n_{1}}-\int_{P \cap \complement A_{n_{1}}}\left\{g(x, u(x))-g\left(x, n_{1}\right)\right\} \mathrm{d} x \\
& >a_{n_{0}}-\frac{a_{n_{0}}}{2} \\
& =\frac{a_{n_{0}}}{2}>0
\end{aligned}
$$

which implies that $G\left(n_{1}\right)=\int_{P} g\left(x, n_{1}\right) \mathrm{d} x>\int_{P} g(x, u(x)) \mathrm{d} x+\frac{a_{n_{0}}}{2}=\frac{a_{n_{0}}}{2}>0$. We can take $u_{0}^{+}=n_{1}$. The second statement follows in a similar way. The last one is a trivial consequence of the previous statements and the continuity of $G$. 


\subsection{Existence and uniqueness of periodic solution for a penalized problem}

For any $\alpha>0$ we consider the modified problem

$$
\alpha u-\Delta u+g(x, u(x))=0, \quad x \in \mathbb{R}^{N} .
$$

We intend to prove the existence and uniqueness of periodic solutions for (9). Under the condition $\left(C_{4}\right)$ we establish uniform estimates for the sequence of penalized solutions $\left(u_{\alpha}\right)_{\alpha>0}$ and finally we conclude by passing to the limit for $\alpha \searrow 0$.

Proposition 2.4. Assume that $g$ satisfies $\left(H_{1}\right),\left(H_{2}\right)$. Then for any $\alpha>0$ there is at most one periodic solution for $(9)$.

Proof. Consider $u, v$ two periodic solutions of (9). By using the weak formulation with the test function $u-v$ one gets

$$
\alpha \int_{P}|u(x)-v(x)|^{2} \mathrm{~d} x+\int_{P}|\nabla u-\nabla v|^{2} \mathrm{~d} x+\int_{P}(g(x, u(x))-g(x, v(x)))(u(x)-v(x)) \mathrm{d} x=0,
$$

and the conclusion follows by the monotonicity of $g$.

For the existence part we regularize the nonlinearity and construct solutions by using the Banach fixed point theorem. We use the following classical results

Lemma 2.1. Assume that $\beta: \mathbb{R} \rightarrow \mathbb{R}$ is a continuous nondecreasing function such that $\beta(0)=0$. We denote by $1: \mathbb{R} \rightarrow \mathbb{R}$ the identity function on $\mathbb{R}$ and for any $\varepsilon>0$ we consider $\beta_{\varepsilon}(u)=\beta\left((1+\varepsilon \beta)^{-1}(u)\right)$, $u \in \mathbb{R}$. Then the following properties hold

1) $(1+\varepsilon \beta)^{-1}$ is nondecreasing, Lipschitz continuous of constant 1 and $(1+\varepsilon \beta)^{-1}(0)=0$;

2) $\beta_{\varepsilon}$ is nondecreasing, Lipschitz continuous of constant $1 / \varepsilon, \beta_{\varepsilon}(0)=0$. In particular $\left|\beta_{\varepsilon}(u)\right| \leq|u| / \varepsilon, \forall u \in \mathbb{R}$;

3) For any $u \in \mathbb{R}$ we have

$$
\left|(1+\varepsilon \beta)^{-1}(u)\right| \leq|u|, \quad\left|\beta_{\varepsilon}(u)\right| \leq|\beta(u)|, \quad \lim _{\varepsilon \searrow 0}(1+\varepsilon \beta)^{-1}(u)=u, \lim _{\varepsilon \searrow 0} \beta_{\varepsilon}(u)=\beta(u) ;
$$

4) For any $u \in \mathbb{R}$ we have $\beta_{\varepsilon}(u)=\frac{1}{\varepsilon}\left(u-(1+\varepsilon \beta)^{-1}(u)\right)$.

Lemma 2.2. Assume that $g: \mathbb{R}^{N} \times \mathbb{R} \rightarrow \mathbb{R}$ is continuous, nondecreasing w.r.t. $u, g(x, 0)=0$ a.e. $x \in \mathbb{R}^{N}$. Let us denote by $g_{\varepsilon}$ the function $g_{\varepsilon}(x, u)=g\left(x,(1+\varepsilon g(x, \cdot))^{-1}(u)\right),(x, u) \in \mathbb{R}^{N} \times \mathbb{R}$.

1) If there is a constant $C$ such that

$$
\left|g\left(x_{1}, u\right)-g\left(x_{2}, u\right)\right| \leq C|u|^{p}\left|x_{1}-x_{2}\right|, \text { a.e. }\left(x_{1}, x_{2}\right) \in \mathbb{R}^{2 N}, \forall u \in \mathbb{R},
$$

then we have

$$
\left|g_{\varepsilon}\left(x_{1}, u\right)-g_{\varepsilon}\left(x_{2}, u\right)\right| \leq 3 C|u|^{p}\left|x_{1}-x_{2}\right|, \text { a.e. }\left(x_{1}, x_{2}\right) \in \mathbb{R}^{2 N}, \forall u \in \mathbb{R} ;
$$

2) if for any $R>0$ there is $C_{R}$ such that

$$
\left|g\left(x_{1}, u\right)-g\left(x_{2}, u\right)\right| \leq C_{R}\left|x_{1}-x_{2}\right|, \quad\left(x_{1}, x_{2}, u\right) \in \mathbb{R}^{N} \times \mathbb{R}^{N} \times[-R, R],
$$

then

$$
\left|g_{\varepsilon}\left(x_{1}, u\right)-g_{\varepsilon}\left(x_{2}, u\right)\right| \leq 3 C_{R}\left|x_{1}-x_{2}\right|, \quad\left(x_{1}, x_{2}, u\right) \in \mathbb{R}^{N} \times \mathbb{R}^{N} \times[-R, R] .
$$

Proof. 1) For $\left(x_{1}, x_{2}, u\right) \in \mathbb{R}^{2 N} \times \mathbb{R}$ we have $g_{\varepsilon}\left(x_{1}, u\right)=g\left(x_{1}, v_{1}\right), g_{\varepsilon}\left(x_{2}, u\right)=g\left(x_{2}, v_{2}\right)$ where $v_{1}+\varepsilon g\left(x_{1}, v_{1}\right)=u$, $v_{2}+\varepsilon g\left(x_{2}, v_{2}\right)=u$ and therefore

$$
\begin{aligned}
\left|g_{\varepsilon}\left(x_{1}, u\right)-g_{\varepsilon}\left(x_{2}, u\right)\right| & \leq\left|g\left(x_{1}, v_{1}\right)-g\left(x_{1}, v_{2}\right)\right|+\left|g\left(x_{1}, v_{2}\right)-g\left(x_{2}, v_{2}\right)\right| \\
& \leq\left|g\left(x_{1}, v_{1}\right)-g\left(x_{1}, v_{2}\right)\right|+C\left|v_{2}\right|^{p}\left|x_{1}-x_{2}\right| .
\end{aligned}
$$


We have also the inequality $\left|v_{2}\right|=\left|\left(1+\varepsilon g\left(x_{2}, \cdot\right)\right)^{-1}(u)\right| \leq|u|$. Note also that

$$
v_{1}-v_{2}+\varepsilon\left(g\left(x_{1}, v_{1}\right)-g\left(x_{1}, v_{2}\right)\right)=-\varepsilon\left(g\left(x_{1}, v_{2}\right)-g\left(x_{2}, v_{2}\right)\right) .
$$

After multiplication by $v_{1}-v_{2}$ one gets

$$
\left|v_{1}-v_{2}\right| \leq \varepsilon C\left|v_{2}\right|^{p}\left|x_{1}-x_{2}\right| \leq \varepsilon C|u|^{p}\left|x_{1}-x_{2}\right| .
$$

We deduce also from (11) that

$$
\left|g\left(x_{1}, v_{1}\right)-g\left(x_{1}, v_{2}\right)\right|=\left|g\left(x_{1}, v_{2}\right)-g\left(x_{2}, v_{2}\right)+\frac{v_{1}-v_{2}}{\varepsilon}\right| \leq 2 C|u|^{p}\left|x_{1}-x_{2}\right| .
$$

Combining (10) and (13) yields the conclusion of the first statement. The second one follows similarly.

Lemma 2.3. Consider $u \in L_{\mathrm{loc}}^{2}\left(\mathbb{R}^{N}\right)$ a $L=\left(L_{1}, L_{2}, \ldots, L_{N}\right)$ periodic function. Then the following statements hold

1) if $u \in H_{\#}^{1}\left(\mathbb{R}^{N}\right)$ then $\|u(\cdot+h)-u(\cdot)\|_{L_{\#}^{2}\left(\mathbb{R}^{N}\right)} \leq\|\nabla u\|_{L_{\#}^{2}\left(\mathbb{R}^{N}\right)}|h|, \quad \forall h \in \mathbb{R}^{N}$;

2) if there is a constant $C$ such that $\|u(\cdot+h)-u(\cdot)\|_{L_{\#}^{2}\left(\mathbb{R}^{N}\right)} \leq C|h|, \quad \forall h \in \mathbb{R}^{N}$, then $u$ belongs to $H_{\#}^{1}\left(\mathbb{R}^{N}\right)$ and we have $\|\nabla u\|_{L_{\#}^{2}\left(\mathbb{R}^{N}\right)} \leq C \sqrt{N}$.

Lemma 2.4. Assume that $\alpha>0, f \in L_{\#}^{2}\left(\mathbb{R}^{N}\right)$. Then there is a unique periodic solution for the linear problem $\alpha u-\Delta u=f(x), \quad x \in \mathbb{R}^{N}$, and we have the estimate $\|u\|_{H_{\#}^{1}\left(\mathbb{R}^{N}\right)} \leq \frac{\|f\|_{L_{\#}^{2}\left(\mathbb{R}^{N}\right)}}{\min \{1, \alpha\}}$.

Proof. Consider the bilinear form $a(u, v)=\alpha \int_{P} u(x) v(x) \mathrm{d} x+\int_{P} \nabla u \cdot \nabla v \mathrm{~d} x, u, v \in H_{\#}^{1}\left(\mathbb{R}^{N}\right)$ and apply the Lax-Milgram lemma.

We prove now the existence of periodic solution for (9).

Theorem 2.1. Assume that $g: \mathbb{R}^{N} \times \mathbb{R} \rightarrow \mathbb{R}$ satisfies $\left(H_{1}\right),\left(H_{2}\right),\left(H_{3}\right),\left(H_{4}\right)$. Then for any $\alpha>0$ there is a unique periodic solution $u_{\alpha} \in H_{\#}^{2}\left(\mathbb{R}^{N}\right)$ for (9) and we have the estimate

$$
\begin{gathered}
\left\|u_{\alpha}\right\|_{H_{\#}^{2}\left(\mathbb{R}^{N}\right)} \leq C(\alpha)\left(\|g(\cdot, 0)\|_{L_{\#}^{2}\left(\mathbb{R}^{N}\right)}+C_{R(\alpha)}\right), \text { if } N=1, \\
\left\|u_{\alpha}\right\|_{H_{\#}^{2}\left(\mathbb{R}^{N}\right)} \leq C(\alpha)\left(\|g(\cdot, 0)\|_{L_{\#}^{2}\left(\mathbb{R}^{N}\right)}+C_{g}\|g(\cdot, 0)\|_{L_{\#}^{2}\left(\mathbb{R}^{N}\right)}^{p}\right), \text { if } N \geq 2, \\
\left\|g\left(\cdot, u_{\alpha}(\cdot)\right)\right\|_{L_{\#}^{2}\left(\mathbb{R}^{N}\right)} \leq\|g(\cdot, 0)\|_{L_{\#}^{2}\left(\mathbb{R}^{N}\right)}+C(\alpha, g, p), \forall N \geq 1 .
\end{gathered}
$$

Proof. The problem (9) can be written $\alpha u-\Delta u+g(x, u(x))-g(x, 0)=-g(x, 0), \quad x \in \mathbb{R}^{N}$, and therefore it is sufficient to study

$$
\alpha u-\Delta u+g(x, u(x))=f(x), \quad x \in \mathbb{R}^{N},
$$

where $g$ satisfies $\left(H_{1}\right),\left(H_{2}\right),\left(H_{3}\right), g(\cdot, 0)=0, f \in L_{\#}^{2}\left(\mathbb{R}^{N}\right)$. For any $\varepsilon>0$ we consider $g_{\varepsilon}(x, u)=g(x,(1+$ $\left.\varepsilon g(x, \cdot))^{-1}(u)\right)$. We define the application $T_{\varepsilon}: L_{\#}^{2}\left(\mathbb{R}^{N}\right) \rightarrow L_{\#}^{2}\left(\mathbb{R}^{N}\right)$ as follows : for any $u \in L_{\#}^{2}\left(\mathbb{R}^{N}\right), T_{\varepsilon}(u)=v$ where $v$ is the unique periodic solution of the problem

$$
\alpha v-\Delta v+\frac{v}{\varepsilon}=\frac{1}{\varepsilon}(1+\varepsilon g(x, \cdot))^{-1}(u(x))+f(x), \quad x \in \mathbb{R}^{N} .
$$

Note that $\varepsilon^{-1}\left|(1+\varepsilon g(x, \cdot))^{-1}(u(x))\right| \leq \varepsilon^{-1}|u(x)| \in L_{\#}^{2}\left(\mathbb{R}^{N}\right)$ and therefore the existence and uniqueness of $v$ follow by Lemma 2.4. We can prove that $T_{\varepsilon}$ is a contraction. Consider $u_{1}, u_{2} \in L_{\#}^{2}\left(\mathbb{R}^{N}\right)$ and denote $v_{1}=T_{\varepsilon}\left(u_{1}\right)$, 
$v_{2}=T_{\varepsilon}\left(v_{2}\right)$. We have

$$
\begin{aligned}
\left(\alpha+\varepsilon^{-1}\right) \int_{P}\left|v_{1}-v_{2}\right|^{2} \mathrm{~d} x & +\int_{P}\left|\nabla v_{1}-\nabla v_{2}\right|^{2} \mathrm{~d} x \\
& =\frac{1}{\varepsilon} \int_{P}\left((1+\varepsilon g(x, \cdot))^{-1} u_{1}-(1+\varepsilon g(x, \cdot))^{-1} u_{2}\right)\left(v_{1}-v_{2}\right) \mathrm{d} x \\
& \leq \frac{1}{\varepsilon} \int_{P}\left(u_{1}(x)-u_{2}(x)\right)\left(v_{1}(x)-v_{2}(x)\right) \mathrm{d} x \\
& \leq \frac{1}{2 \varepsilon} \int_{P}\left|u_{1}-u_{2}\right|^{2} \mathrm{~d} x+\frac{1}{2 \varepsilon} \int_{P}\left|v_{1}-v_{2}\right|^{2} \mathrm{~d} x
\end{aligned}
$$

and therefore we obtain

$$
\left(\alpha+\frac{1}{2 \varepsilon}\right)\left\|v_{1}-v_{2}\right\|_{L_{\#}^{2}\left(\mathbb{R}^{N}\right)}^{2} \leq \frac{1}{2 \varepsilon}\left\|u_{1}-u_{2}\right\|_{L_{\#}^{2}\left(\mathbb{R}^{N}\right)}^{2},
$$

which implies that $T_{\varepsilon}$ is a contraction of constant $(1+2 \varepsilon \alpha)^{-1 / 2}$. By the Banach fixed point theorem we deduce that there is a unique $u_{\varepsilon} \in L_{\#}^{2}\left(\mathbb{R}^{N}\right)$ solution of

$$
\alpha u_{\varepsilon}-\Delta u_{\varepsilon}+g_{\varepsilon}\left(x, u_{\varepsilon}(x)\right)=f(x), \quad x \in \mathbb{R}^{N}
$$

We intend to pass to the limit for $\varepsilon \searrow 0$ and for this we are looking for uniform estimates w.r.t. $\varepsilon$. Since $g_{\varepsilon}(x, u) u \geq 0,(x, u) \in \mathbb{R}^{N} \times \mathbb{R}$ we obtain

$$
\alpha \int_{P}\left|u_{\varepsilon}\right|^{2} \mathrm{~d} x+\int_{P}\left|\nabla u_{\varepsilon}\right|^{2} \mathrm{~d} x \leq \int_{P} f u_{\varepsilon} \mathrm{d} x
$$

and therefore we deduce that $\left\|u_{\varepsilon}\right\|_{H_{\#}^{1}\left(\mathbb{R}^{N}\right)} \leq \frac{\|f\|_{L_{\#}^{2}\left(\mathbb{R}^{N}\right)}}{\min \{1, \alpha\}}, \forall \varepsilon>0$. In particular, if $N=1$ the sequence $\left(u_{\varepsilon}\right)_{\varepsilon}$ is uniformly bounded

$$
\left\|u_{\varepsilon}\right\|_{L^{\infty}} \leq C\left\|u_{\varepsilon}\right\|_{H_{\#}^{1}(\mathbb{R})} \leq C \frac{\|f\|_{L_{\#}^{2}(\mathbb{R})}}{\min \{1, \alpha\}}, \quad \forall \varepsilon>0 .
$$

We introduce the notation $D_{h} v(x)=v(x+h)-v(x),(x, h) \in \mathbb{R}^{2 N}$. We have for any $h \in \mathbb{R}^{N}$

$$
\begin{aligned}
\alpha D_{h} u_{\varepsilon}(x)-\Delta D_{h} u_{\varepsilon}(x) & +g_{\varepsilon}\left(x+h, u_{\varepsilon}(x+h)\right)-g_{\varepsilon}\left(x+h, u_{\varepsilon}(x)\right) \\
& =g_{\varepsilon}\left(x, u_{\varepsilon}(x)\right)-g_{\varepsilon}\left(x+h, u_{\varepsilon}(x)\right)+D_{h} f(x) .
\end{aligned}
$$

After multiplication by $D_{h} u_{\varepsilon}(x)$ we obtain

$$
\begin{aligned}
\alpha \int_{P}\left|D_{h} u_{\varepsilon}(x)\right|^{2} \mathrm{~d} x+\int_{P}\left|\nabla D_{h} u_{\varepsilon}\right|^{2} \mathrm{~d} x & \leq \mathbf{1}_{\{N=1\}} \int_{P} 3 C_{R}|h|\left|D_{h} u_{\varepsilon}(x)\right| \mathrm{d} x \\
& +\mathbf{1}_{\{N \geq 2\}} \int_{P} 3 C_{g}|h|\left|u_{\varepsilon}(x)\right|^{p}\left|D_{h} u_{\varepsilon}(x)\right| \mathrm{d} x \\
& +\int_{P} D_{h} f(x) D_{h} u_{\varepsilon}(x) \mathrm{d} x,
\end{aligned}
$$

where $R=R(\alpha)=\sup _{\varepsilon>0}\left\|u_{\varepsilon}\right\|_{L^{\infty}}$ if $N=1$. By periodicity we can write

$$
\begin{aligned}
\int_{P} D_{h} f(x) D_{h} u_{\varepsilon}(x) \mathrm{d} x & =-\int_{P} f(x)\left(D_{h} u_{\varepsilon}(x)-D_{h} u_{\varepsilon}(x-h)\right) \mathrm{d} x \\
& \leq\|f\|_{L_{\#}^{2}\left(\mathbb{R}^{N}\right)}|h|\left\|D_{h} \nabla u_{\varepsilon}\right\|_{L_{\#}^{2}\left(\mathbb{R}^{N}\right)} .
\end{aligned}
$$


If $N \geq 2$ by Sobolev inequalities we have

$$
\left\|\left|u_{\varepsilon}\right|^{p}\right\|_{L^{\frac{p^{\star}}{p}}(P)} \leq C\left\|u_{\varepsilon}\right\|_{H_{\#}^{1}\left(\mathbb{R}^{N}\right)}^{p} \leq C(\alpha)\|f\|_{L_{\#}^{2}\left(\mathbb{R}^{N}\right)}^{p}, \forall \varepsilon>0,
$$

and

$$
\left\|D_{h} u_{\varepsilon}\right\|_{L^{p^{\star}}(P)} \leq C\left\{\left\|D_{h} u_{\varepsilon}\right\|_{L_{\#}^{2}\left(\mathbb{R}^{N}\right)}+\left\|D_{h} \nabla u_{\varepsilon}\right\|_{L_{\#}^{2}\left(\mathbb{R}^{N}\right)}\right\},
$$

for any $p^{\star} \geq p$ if $N=2$ and $p^{\star}=\frac{2 N}{N-2}$ if $N \geq 3$. Consider $q$ given by $\frac{1}{q}=\frac{p}{p^{\star}}+\frac{1}{p^{\star}}$. If $N=2$ we take $p^{\star} \geq p+1$ such that $q \geq 1$. If $N \geq 3$ by the hypothesis $\left(H_{3}\right)$ we have $p+1 \leq \frac{N+2}{N-2}+1=\frac{2 N}{N-2}=p^{\star}$ and we also have $q \geq 1$. By Holder inequality we obtain for $N \geq 2$

$$
\begin{aligned}
\int_{P}\left|u_{\varepsilon}(x)\right|^{p}\left|D_{h} u_{\varepsilon}(x)\right| \mathrm{d} x & \leq C\left\|\left|u_{\varepsilon}\right|^{p}\left|D_{h} u_{\varepsilon}\right|\right\|_{L^{q}(P)} \\
& \leq C\left\|\left|u_{\varepsilon}\right|^{p}\right\|_{L^{\frac{p^{\star}}{p}}(P)}\left\|D_{h} u_{\varepsilon}\right\|_{L^{p^{\star}}(P)} \\
& \leq C\left\|u_{\varepsilon}\right\|_{H_{\#}^{1}\left(\mathbb{R}^{N}\right)}^{p}\left(\left\|D_{h} u_{\varepsilon}\right\|_{L_{\#}^{2}\left(\mathbb{R}^{N}\right)}+\left\|D_{h} \nabla u_{\varepsilon}\right\|_{L_{\#}^{2}\left(\mathbb{R}^{N}\right)}\right) \\
& \leq C\left\|u_{\varepsilon}\right\|_{H_{\#}^{1}\left(\mathbb{R}^{N}\right)}^{p}\left(|h|\left\|\nabla u_{\varepsilon}\right\|_{L_{\#}^{2}\left(\mathbb{R}^{N}\right)}+\left\|D_{h} \nabla u_{\varepsilon}\right\|_{L_{\#}^{2}\left(\mathbb{R}^{N}\right)}\right) \\
& \leq C(\alpha)|h|\|f\|_{L_{\#}^{2}\left(\mathbb{R}^{N}\right)}^{p}\left(\|f\|_{L_{\#}^{2}\left(\mathbb{R}^{N}\right)}+\frac{\left.\left\|D_{h} \nabla u_{\varepsilon}\right\|_{L_{\#}^{2}\left(\mathbb{R}^{N}\right)}\right) .}{|h|}\right)
\end{aligned}
$$

Combining (21), (22) and (23) we deduce

$$
\begin{aligned}
\frac{\left\|D_{h} \nabla u_{\varepsilon}\right\|_{L_{\#}^{2}\left(\mathbb{R}^{N}\right)}^{2}}{|h|^{2}} & \leq C_{g} C(\alpha)\|f\|_{L_{\#}^{2}\left(\mathbb{R}^{N}\right)}^{p}\left(\|f\|_{L_{\#}^{2}\left(\mathbb{R}^{N}\right)}+\frac{\left\|D_{h} \nabla u_{\varepsilon}\right\|_{L_{\#}^{2}\left(\mathbb{R}^{N}\right)}}{|h|}\right) \\
& +\|f\|_{L_{\#}^{2}\left(\mathbb{R}^{N}\right)} \frac{\left\|D_{h} \nabla u_{\varepsilon}\right\|_{L_{\#}^{2}\left(\mathbb{R}^{N}\right)}}{|h|},
\end{aligned}
$$

which implies that $\left\|D_{h} \nabla u_{\varepsilon}\right\|_{L_{\#}^{2}\left(\mathbb{R}^{N}\right)} \leq C(\alpha)|h|\left(\|f\|_{L_{\#}^{2}\left(\mathbb{R}^{N}\right)}+C_{g}\|f\|_{L_{\#}^{2}\left(\mathbb{R}^{N}\right)}^{p}\right)$. By Lemma 2.3 one gets that $\nabla u_{\varepsilon} \in H_{\#}^{1}\left(\mathbb{R}^{N}\right)$ and

$$
\left\|u_{\varepsilon}\right\|_{H_{\#}^{2}\left(\mathbb{R}^{N}\right)} \leq C(\alpha)\left(\|f\|_{L_{\#}^{2}\left(\mathbb{R}^{N}\right)}+C_{g}\|f\|_{L_{\#}^{2}\left(\mathbb{R}^{N}\right)}^{p}\right)
$$

In the case $N=1$ we obtain from $(21)$ and $(22)$

$$
\frac{\left\|D_{h} \nabla u_{\varepsilon}\right\|_{L_{\#}^{2}(\mathbb{R})}^{2}}{|h|^{2}} \leq\|f\|_{L_{\#}^{2}(\mathbb{R})}\left(C(\alpha) C_{R}+\frac{\left\|D_{h} \nabla u_{\varepsilon}\right\|_{L_{\#}^{2}(\mathbb{R})}}{|h|}\right)
$$

and therefore one gets

$$
\frac{\left\|D_{h} \nabla u_{\varepsilon}\right\|_{L_{\#}^{2}(\mathbb{R})}}{|h|} \leq\|f\|_{L_{\#}^{2}(\mathbb{R})}+\left(C(\alpha) C_{R}\|f\|_{L_{\#}^{2}(\mathbb{R})}\right)^{\frac{1}{2}} \leq \frac{3}{2}\|f\|_{L_{\#}^{2}(\mathbb{R})}+\frac{1}{2} C(\alpha) C_{R} .
$$

In this case we obtain $\left\|u_{\varepsilon}\right\|_{H_{\#}^{2}(\mathbb{R})} \leq C(\alpha)\left(\|f\|_{L_{\#}^{2}(\mathbb{R})}+C_{R}\right), \forall \varepsilon>0$. We establish also an estimate for the $L_{\#}^{2}\left(\mathbb{R}^{N}\right)$ norm of $g_{\varepsilon}\left(\cdot, u_{\varepsilon}(\cdot)\right)$. We want to multiply $(20)$ by $g_{\varepsilon}\left(\cdot, u_{\varepsilon}(\cdot)\right)$. Note that this function belongs to $H_{\#}^{1}\left(\mathbb{R}^{N}\right)$. Indeed, we have $\left|g_{\varepsilon}\left(\cdot, u_{\varepsilon}(\cdot)\right)\right| \leq \varepsilon^{-1}\left|u_{\varepsilon}\right| \in L_{\#}^{2}\left(\mathbb{R}^{N}\right)$ and

$$
\begin{aligned}
\left|\nabla_{x}\left\{g_{\varepsilon}\left(x, u_{\varepsilon}(x)\right)\right\}\right| & =\left|\left(\nabla_{x} g_{\varepsilon}\right)\left(x, u_{\varepsilon}(x)\right)+\partial_{u} g_{\varepsilon}\left(x, u_{\varepsilon}(x)\right) \nabla u_{\varepsilon}\right| \\
& \leq\left|\left(\nabla_{x} g_{\varepsilon}\right)\left(x, u_{\varepsilon}(x)\right)\right|+\frac{1}{\varepsilon}\left|\nabla u_{\varepsilon}\right| .
\end{aligned}
$$


We need to check that $\left(\nabla_{x} g_{\varepsilon}\right)\left(\cdot, u_{\varepsilon}(\cdot)\right)$ belongs to $L_{\#}^{2}\left(\mathbb{R}^{N}\right)$. If $N=1$ then $\left(u_{\varepsilon}\right)_{\varepsilon}$ is bounded and by Lemma 2.2 we deduce that $\left(\left(\nabla_{x} g_{\varepsilon}\right)\left(\cdot, u_{\varepsilon}(\cdot)\right)\right)_{\varepsilon}$ is bounded too

$$
\left|\left(\nabla_{x} g_{\varepsilon}\right)\left(x, u_{\varepsilon}(x)\right)\right| \leq 3 C_{R}, \forall \varepsilon>0 \text {, a.e. } x \in \mathbb{R}^{N},
$$

where $R=\sup _{\varepsilon>0}\left\|u_{\varepsilon}\right\|_{L^{\infty}}$. If $N \geq 2$ we know that

$$
\begin{aligned}
\int_{P}\left|\nabla_{x} g_{\varepsilon}\right|^{2}\left(x, u_{\varepsilon}(x)\right) \mathrm{d} x & \leq \int_{P}\left(3 C_{g}\left|u_{\varepsilon}(x)\right|^{p}\right)^{2} \mathrm{~d} x \leq 9 C_{g}^{2} C\left\|u_{\varepsilon}\right\|_{H_{\#}^{2}\left(\mathbb{R}^{N}\right)}^{2 p} \\
& \leq C_{g}^{2} C(\alpha)\left(\|f\|_{L_{\#}^{2}\left(\mathbb{R}^{N}\right)}+C_{g}\|f\|_{L_{\#}^{2}\left(\mathbb{R}^{N}\right)}^{p}\right)^{2 p} .
\end{aligned}
$$

In the above inequalities we have used the Sobolev inclusion $H_{\#}^{2}\left(\mathbb{R}^{N}\right) \subset L_{\#}^{q}\left(\mathbb{R}^{N}\right)$ with $1 \leq q<+\infty$ if $N \in$ $\{2,3,4\}$ and $1 \leq q \leq \frac{2 N}{N-4}$ if $N>4$. Note that if $N>4$ then $2 p \leq 2 \frac{N+2}{N-2}<\frac{2 N}{N-4}$. After multiplication of (20) by $g_{\varepsilon}\left(\cdot, u_{\varepsilon}(\cdot)\right)$ we obtain

$$
\begin{aligned}
\alpha \int_{P} u_{\varepsilon}(x) g_{\varepsilon}\left(x, u_{\varepsilon}(x)\right) \mathrm{d} x & +\int_{P} \sum_{i=1}^{N} \partial_{x_{i}} u_{\varepsilon}\left(\partial_{x_{i}} g_{\varepsilon}+\partial_{u} g_{\varepsilon} \partial_{x_{i}} u_{\varepsilon}\right) \mathrm{d} x+\int_{P}\left|g_{\varepsilon}\left(x, u_{\varepsilon}(x)\right)\right|^{2} \mathrm{~d} x \\
& =\int_{P} f(x) g_{\varepsilon}\left(x, u_{\varepsilon}(x)\right) \mathrm{d} x
\end{aligned}
$$

Observe that $\int_{P} u_{\varepsilon}(x) g_{\varepsilon}\left(x, u_{\varepsilon}(x)\right) \mathrm{d} x \geq 0, \int_{P} \sum_{i=1}^{N}\left|\partial_{x_{i}} u_{\varepsilon}\right|^{2} \partial_{u} g_{\varepsilon} \mathrm{d} x \geq 0$ and therefore we deduce

$$
\begin{aligned}
\int_{P}\left|g_{\varepsilon}\left(x, u_{\varepsilon}(x)\right)\right|^{2} \mathrm{~d} x & \leq\left(\int_{P}\left|\nabla u_{\varepsilon}\right|^{2} \mathrm{~d} x\right)^{\frac{1}{2}}\left(\int_{P}\left|\nabla_{x} g_{\varepsilon}\right|^{2}\left(x, u_{\varepsilon}(x)\right) \mathrm{d} x\right)^{\frac{1}{2}} \\
& +\left(\int_{P}|f(x)|^{2} \mathrm{~d} x\right)^{\frac{1}{2}}\left(\int_{P}\left|g_{\varepsilon}\left(x, u_{\varepsilon}(x)\right)\right|^{2} \mathrm{~d} x\right)^{\frac{1}{2}} .
\end{aligned}
$$

Finally one gets from (25), (26) and (27)

$$
\left(\int_{P}\left|g_{\varepsilon}\left(x, u_{\varepsilon}(x)\right)\right|^{2} \mathrm{~d} x\right)^{\frac{1}{2}} \leq\|f\|_{L_{\#}^{2}\left(\mathbb{R}^{N}\right)}+C(\alpha, f, g, p), \quad \forall \varepsilon>0 .
$$

We intend to prove that $\left(u_{\varepsilon}\right)_{\varepsilon}$ converges in $H_{\#}^{1}\left(\mathbb{R}^{N}\right)$. The arguments are standard. For any $\varepsilon, \lambda>0$ we have

$$
\alpha\left(u_{\varepsilon}-u_{\lambda}\right)-\Delta\left(u_{\varepsilon}-u_{\lambda}\right)+g_{\varepsilon}\left(x, u_{\varepsilon}(x)\right)-g_{\lambda}\left(x, u_{\lambda}(x)\right)=0, \quad x \in \mathbb{R}^{N},
$$

and therefore one gets

$$
\begin{aligned}
\alpha \int_{P}\left|u_{\varepsilon}-u_{\lambda}\right|^{2} \mathrm{~d} x & +\int_{P}\left|\nabla u_{\varepsilon}-\nabla u_{\lambda}\right|^{2} \mathrm{~d} x+\int_{P}\left[g_{\varepsilon}\left(x, u_{\varepsilon}(x)\right)-g_{\lambda}\left(x, u_{\lambda}(x)\right)\right]\left[u_{\varepsilon}-u_{\lambda}\right] \mathrm{d} x \\
& =0 .
\end{aligned}
$$

Since for any $v$ we have $v=(1+\varepsilon g(x, \cdot))^{-1}(v)+\varepsilon g_{\varepsilon}(x, v)$ we can write

$$
\begin{aligned}
\int_{P}\left[g_{\varepsilon}\left(x, u_{\varepsilon}(x)\right)\right. & \left.-g_{\lambda}\left(x, u_{\lambda}(x)\right)\right]\left[u_{\varepsilon}-u_{\lambda}\right] \mathrm{d} x=\int_{P}\left[(1+\varepsilon g(x, \cdot))^{-1} u_{\varepsilon}-(1+\lambda g(x, \cdot))^{-1} u_{\lambda}\right] \\
& \times\left[g\left(x,(1+\varepsilon g(x, \cdot))^{-1} u_{\varepsilon}(x)\right)-g\left(x,(1+\lambda g(x, \cdot))^{-1} u_{\lambda}(x)\right)\right] \mathrm{d} x \\
& +\int_{P}\left[g_{\varepsilon}\left(x, u_{\varepsilon}(x)\right)-g_{\lambda}\left(x, u_{\lambda}(x)\right)\right]\left[\varepsilon g_{\varepsilon}\left(x, u_{\varepsilon}(x)\right)-\lambda g_{\lambda}\left(x, u_{\lambda}(x)\right)\right] \mathrm{d} x .
\end{aligned}
$$


Combining (28) and (29) yields

$$
\alpha \int_{P}\left|u_{\varepsilon}-u_{\lambda}\right|^{2} \mathrm{~d} x+\int_{P}\left|\nabla u_{\varepsilon}-\nabla u_{\lambda}\right|^{2} \mathrm{~d} x \leq 2(\varepsilon+\lambda) \sup _{\mu>0} \int_{P}\left|g_{\mu}\left(x, u_{\mu}(x)\right)\right|^{2} \mathrm{~d} x,
$$

and therefore $\left(u_{\varepsilon}\right)_{\varepsilon}$ is a Cauchy sequence in $H_{\#}^{1}\left(\mathbb{R}^{N}\right)$. Denote $u=\lim _{\varepsilon \backslash 0} u_{\varepsilon}$ in $H_{\#}^{1}\left(\mathbb{R}^{N}\right)$. Since $\left(g_{\varepsilon}\left(\cdot, u_{\varepsilon}(\cdot)\right)\right)_{\varepsilon}$ is bounded in $L_{\#}^{2}\left(\mathbb{R}^{N}\right)$ we can extract a sequence $\left(\varepsilon_{k}\right)_{k}, \lim _{k \rightarrow+\infty} \varepsilon_{k}=0$, such that $\left(g_{\varepsilon_{k}}\left(\cdot, u_{\varepsilon_{k}}(\cdot)\right)\right)_{k}$ converges weakly to some function $z$ in $L_{\#}^{2}\left(\mathbb{R}^{N}\right)$. We have the strong convergence in $L_{\#}^{2}\left(\mathbb{R}^{N}\right)$

$$
u-(1+\varepsilon g(x, \cdot))^{-1}\left(u_{\varepsilon}\right)=\varepsilon g_{\varepsilon}\left(x, u_{\varepsilon}\right)+u-u_{\varepsilon} \rightarrow 0 .
$$

Thus, after extracting a subsequence if necessary, we have

$$
\lim _{k \rightarrow+\infty}\left(1+\varepsilon_{k} g(x, \cdot)\right)^{-1}\left(u_{\varepsilon_{k}}(x)\right)=u(x), \quad \lim _{k \rightarrow+\infty} g_{\varepsilon_{k}}\left(x, u_{\varepsilon_{k}}(x)\right)=g(x, u(x)) \text {, a.e. } x \in \mathbb{R}^{N} .
$$

We intend to prove that $z(x)=g(x, u(x))$ a.e. $x \in \mathbb{R}^{N}$. By Fatou lemma we obtain

$$
\int_{P}|g(x, u(x))|^{2} \mathrm{~d} x \leq \liminf _{k \rightarrow+\infty} \int_{P}\left|g_{\varepsilon_{k}}\left(x, u_{\varepsilon_{k}}(x)\right)\right|^{2} \mathrm{~d} x \leq \sup _{\varepsilon>0} \int_{P}\left|g_{\varepsilon}\left(x, u_{\varepsilon}(x)\right)\right|^{2} \mathrm{~d} x,
$$

and thus $g(\cdot, u(\cdot))$ belongs to $L_{\#}^{2}\left(\mathbb{R}^{N}\right)$. Pick $\varphi \in L_{\#}^{2}\left(\mathbb{R}^{N}\right)$ and $\eta>0$. By using the Egorov theorem there is a measurable set $E_{\eta} \subset P$ such that $\left(g_{\varepsilon_{k}}\left(\cdot, u_{\varepsilon_{k}}(\cdot)\right)\right)_{k}$ converges uniformly on $E_{\eta}$ towards $g(\cdot, u(\cdot))$ and meas $(P \cap$ $\left.\complement E_{\eta}\right)<\eta$. For any $\delta>0$ there is $k_{\delta}$ such that $\left|g_{k}(x)-g(x)\right| \leq \delta \forall k \geq k_{\delta}, x \in E_{\eta}$ and therefore we have for $k \geq k_{\delta}$

$$
\begin{aligned}
\left|\int_{P}\left[g_{\varepsilon_{k}}\left(x, u_{\varepsilon_{k}}(x)\right)-g(x, u(x))\right] \varphi(x) \mathrm{d} x\right| & \leq\left|\int_{E_{\eta}}\left[g_{\varepsilon_{k}}\left(x, u_{\varepsilon_{k}}(x)\right)-g(x, u(x))\right] \varphi(x) \mathrm{d} x\right| \\
& +\left|\int_{P \cap C E_{\eta}}\left[g_{\varepsilon_{k}}\left(x, u_{\varepsilon_{k}}(x)\right)-g(x, u(x))\right] \varphi(x) \mathrm{d} x\right| \\
& \leq\left(\left\|g_{\varepsilon_{k}}\left(\cdot, u_{\varepsilon_{k}}(\cdot)\right)\right\|_{L_{\#}^{2}\left(\mathbb{R}^{N}\right)}+\|g(\cdot, u(\cdot))\|_{L_{\#}^{2}\left(\mathbb{R}^{N}\right)}\right) \\
& \times\|\varphi\|_{L^{2}\left(P \cap C E_{\eta}\right)}+\delta \int_{P}|\varphi(x)| \mathrm{d} x .
\end{aligned}
$$

By passing $k \rightarrow+\infty$ we find that for any $\eta>0, \delta>0$

$$
\left|\int_{P}[z(x)-g(x, u(x))] \varphi(x) \mathrm{d} x\right| \leq \delta \int_{P}|\varphi(x)| \mathrm{d} x+2 \sup _{\varepsilon>0}\left\|g_{\varepsilon}\left(\cdot, u_{\varepsilon}(\cdot)\right)\right\|_{L_{\#}^{2}\left(\mathbb{R}^{N}\right)}\|\varphi\|_{L^{2}\left(P \cap C E_{\eta}\right)} .
$$

By letting first $\delta \searrow 0$ and then $\eta \searrow 0$ we deduce that $\int_{P}[z(x)-g(x, u(x))] \varphi(x) \mathrm{d} x=0, \forall \varphi \in L_{\#}^{2}\left(\mathbb{R}^{N}\right)$ which implies that $z(x)=g(x, u(x))$ a.e. $x \in \mathbb{R}^{N}$. Now we obtain easily that $u$ is a solution for (17). Indeed, for any $v \in H_{\#}^{1}\left(\mathbb{R}^{N}\right)$ we write

$$
\alpha \int_{P} u_{\varepsilon_{k}}(x) v(x) \mathrm{d} x+\int_{P} \nabla u_{\varepsilon_{k}} \nabla v \mathrm{~d} x+\int_{P} g_{\varepsilon_{k}}\left(x, u_{\varepsilon_{k}}(x)\right) v(x) \mathrm{d} x=\int_{P} f(x) v(x) \mathrm{d} x,
$$

and by passing to the limit for $k \rightarrow+\infty$ one gets

$$
\alpha \int_{P} u(x) v(x) \mathrm{d} x+\int_{P} \nabla u \nabla v \mathrm{~d} x+\int_{P} g(x, u(x)) v(x) \mathrm{d} x=\int_{P} f(x) v(x) \mathrm{d} x, \forall v \in H_{\#}^{1}\left(\mathbb{R}^{N}\right) .
$$


Moreover $u$ belongs to $H_{\#}^{2}\left(\mathbb{R}^{N}\right)$ and verifies the estimate (14) if $N=1$, (15) if $N \geq 2$ and (16) for any $N \geq 1$.

Remark 2.2. If $g(x, u)=\beta(u)-f(x)$, with $\beta: \mathbb{R} \rightarrow \mathbb{R}$ continuous, nondecreasing, $\beta(0)=0, f \in L_{\#}^{2}\left(\mathbb{R}^{N}\right)$, the above proof guarantees the existence of a unique periodic solution $u_{\alpha} \in H_{\#}^{2}\left(\mathbb{R}^{N}\right)$ for $\alpha u-\Delta u+\beta(u)=f(x)$, $x \in \mathbb{R}^{N}$, satisfying

$$
\left\|u_{\alpha}\right\|_{H_{\#}^{1}\left(\mathbb{R}^{N}\right)} \leq \frac{\|f\|_{L_{\#}^{2}\left(\mathbb{R}^{N}\right)}}{\min \{1, \alpha\}},\left\|\beta\left(u_{\alpha}\right)\right\|_{L_{\#}^{2}\left(\mathbb{R}^{N}\right)} \leq\|f\|_{L_{\#}^{2}\left(\mathbb{R}^{N}\right)},
$$

and

$$
\left\|D^{2} u_{\alpha}\right\|_{L_{\#}^{2}\left(\mathbb{R}^{N}\right)}:=\left(\sum_{1 \leq i, j \leq N} \int_{P}\left|\partial_{x_{i} x_{j}}^{2} u_{\alpha}\right|^{2} \mathrm{~d} x\right)^{\frac{1}{2}} \leq C\|f\|_{L_{\#}^{2}\left(\mathbb{R}^{N}\right)}
$$

where $C$ does not depend on $\alpha$.

For other details on nonlinear elliptic equations one can refer to $[3,8,10]$.

Remark 2.3. It is also possible to prove the existence and uniqueness of the periodic solution for (9) by minimizing the strictly convex functional

$$
J_{\alpha}(v)=\frac{\alpha}{2} \int_{P}|v(x)|^{2} \mathrm{~d} x+\frac{1}{2} \int_{P}|\nabla v|^{2} \mathrm{~d} x+\int_{P} F(x, v(x)) \mathrm{d} x,
$$

where $F(x, v)=\int_{0}^{v} g(x, u) \mathrm{d} u,(x, v) \in \mathbb{R}^{N} \times \mathbb{R}$.

\subsection{Estimates for the penalized solutions}

In this paragraph we will use several times the following easy lemma

Lemma 2.5. For any function $v \in H_{\#}^{1}\left(\mathbb{R}^{N}\right)$ we have the inequality

$$
\int_{P} \int_{P}|v(x)-v(y)|^{2} \mathrm{~d} y \mathrm{~d} x \leq \operatorname{meas}(P)\left(\sum_{k=1}^{N} L_{k}^{2}\right) \int_{P}|\nabla v|^{2} \mathrm{~d} x .
$$

Proof. The arguments are standard. Without loss of generality we can assume that $v \in C_{\#}^{1}\left(\mathbb{R}^{N}\right)$. We have

$$
\begin{aligned}
w(x, y) & :=v(x)-v(y)=v\left(x_{1}, x_{2}, \ldots, x_{N}\right)-v\left(y_{1}, x_{2}, \ldots, x_{N}\right) \\
& +v\left(y_{1}, x_{2}, \ldots, x_{N}\right)-v\left(y_{1}, y_{2}, \ldots, x_{N}\right)+\ldots \\
& +v\left(y_{1}, y_{2}, \ldots, x_{N}\right)-v\left(y_{1}, y_{2}, \ldots, y_{N}\right) \\
& =w_{1}(x, y)+w_{2}(x, y)+\ldots+w_{N}(x, y), \quad(x, y) \in \mathbb{R}^{2 N}
\end{aligned}
$$

and therefore $\|w\|_{L_{\#}^{2}\left(\mathbb{R}^{2 N}\right)} \leq\left\|w_{1}\right\|_{L_{\#}^{2}\left(\mathbb{R}^{2 N}\right)}+\left\|w_{2}\right\|_{L_{\#}^{2}\left(\mathbb{R}^{2 N}\right)}+\ldots+\left\|w_{N}\right\|_{L_{\#}^{2}\left(\mathbb{R}^{2 N}\right)}$. Observe that $\left|w_{1}(x, y)\right| \leq$ $\int_{0}^{L_{1}}\left|\partial_{x_{1}} v\left(z, x_{2}, \ldots, x_{N}\right)\right| \mathrm{d} z$ and we deduce that

$$
\int_{P} \int_{P}\left|w_{1}(x, y)\right|^{2} \mathrm{~d} y \mathrm{~d} x \leq \operatorname{meas}(P) L_{1}^{2} \int_{P}\left|\partial_{x_{1}} v\right|^{2} \mathrm{~d} x .
$$

Similarly one gets

$$
\int_{P} \int_{P}\left|w_{k}(x, y)\right|^{2} \mathrm{~d} y \mathrm{~d} x \leq \operatorname{meas}(P) L_{k}^{2} \int_{P}\left|\partial_{x_{k}} v\right|^{2} \mathrm{~d} x, 2 \leq k \leq N,
$$

and the conclusion follows easily. 
Remark 2.4. We recall also the Poincaré inequality

$$
\|v-\langle v\rangle\|_{L_{\#}^{2}\left(\mathbb{R}^{N}\right)} \leq|L|\|\nabla v\|_{L_{\#}^{2}\left(\mathbb{R}^{N}\right)}, \quad \forall v \in H_{\#}^{1}\left(\mathbb{R}^{N}\right),
$$

where $\langle v\rangle=(\operatorname{meas}(P))^{-1} \int_{P} v(x) \mathrm{d} x$ and $|L|=\left(\sum_{k=1}^{N} L_{k}^{2}\right)^{1 / 2}$.

Proposition 2.5. Assume that $g: \mathbb{R}^{N} \times \mathbb{R} \rightarrow \mathbb{R}$ satisfies $\left(H_{1}\right),\left(H_{2}\right),\left(H_{3}\right),\left(H_{4}\right)$ and for any $\alpha>0$ denote by $u_{\alpha}$ the unique periodic solution of $(9)$. We denote by $G$ the function $G(\cdot)=\int_{P} g(x, \cdot) \mathrm{d} x$ and suppose also that

$$
\left(C_{4}\right) \quad 0 \in \operatorname{Int}(\operatorname{Range}(G))
$$

Then there is a constant $C$ not depending on $\alpha$ such that

$$
\left\|u_{\alpha}\right\|_{H_{\#}^{2}\left(\mathbb{R}^{N}\right)}+\left\|g\left(\cdot, u_{\alpha}(\cdot)\right)\right\|_{L_{\#}^{2}\left(\mathbb{R}^{N}\right)} \leq C, \forall 0<\alpha \leq 1
$$

Proof. For any $u_{0} \in \mathbb{R}$ such that $G\left(u_{0}\right)=0$ we can write

$$
\begin{aligned}
\alpha \int_{P} u_{\alpha}(x)\left(u_{\alpha}(x)-u_{0}\right) \mathrm{d} x & +\int_{P}\left|\nabla u_{\alpha}\right|^{2} \mathrm{~d} x+\int_{P}\left[g\left(x, u_{\alpha}(x)\right)-g\left(x, u_{0}\right)\right]\left[u_{\alpha}(x)-u_{0}\right] \mathrm{d} x \\
& =-\int_{P} g\left(x, u_{0}\right)\left(u_{\alpha}(x)-u_{0}\right) \mathrm{d} x
\end{aligned}
$$

Taking into account that $u_{\alpha}\left(u_{\alpha}-u_{0}\right) \geq \frac{1}{2}\left|u_{\alpha}\right|^{2}-\frac{1}{2}\left|u_{0}\right|^{2}$ we obtain the inequality

$$
\begin{aligned}
\frac{\alpha}{2} \int_{P}\left|u_{\alpha}(x)\right|^{2} \mathrm{~d} x & +\int_{P}\left|\nabla u_{\alpha}\right|^{2} \mathrm{~d} x+\int_{P}\left[g\left(x, u_{\alpha}(x)\right)-g\left(x, u_{0}\right)\right]\left[u_{\alpha}(x)-u_{0}\right] \mathrm{d} x \\
& \leq \frac{\alpha}{2} \operatorname{meas}(P)\left|u_{0}\right|^{2}-\int_{P} g\left(x, u_{0}\right)\left(u_{\alpha}(x)-u_{0}\right) \mathrm{d} x .
\end{aligned}
$$

Observe that the last integral can be estimated in the following manner, by using (31)

$$
\begin{aligned}
I & :=-\int_{P} g\left(x, u_{0}\right)\left(u_{\alpha}(x)-u_{0}\right) \mathrm{d} x \\
& =-\frac{1}{\operatorname{meas}(P)} \int_{P} \int_{P}\left[g\left(x, u_{0}\right)-g\left(y, u_{0}\right)\right]\left[u_{\alpha}(x)-u_{0}\right] \mathrm{d} y \mathrm{~d} x \\
& =-\frac{1}{\operatorname{meas}(P)} \int_{P} \int_{P} g\left(x, u_{0}\right)\left(u_{\alpha}(x)-u_{\alpha}(y)\right) \mathrm{d} y \mathrm{~d} x \\
& \leq \frac{1}{\operatorname{meas}(P)}\left(\int_{P} \int_{P}\left|g\left(x, u_{0}\right)\right|^{2} \mathrm{~d} y \mathrm{~d} x\right)^{\frac{1}{2}}\left(\int_{P} \int_{P}\left|u_{\alpha}(x)-u_{\alpha}(y)\right|^{2} \mathrm{~d} y \mathrm{~d} x\right)^{\frac{1}{2}} \\
& \leq|L|\left\|g\left(\cdot, u_{0}\right)\right\|_{L_{\#}^{2}\left(\mathbb{R}^{N}\right)}\left\|\nabla u_{\alpha}\right\|_{L_{\#}^{2}\left(\mathbb{R}^{N}\right)}
\end{aligned}
$$

From (32) and (33) we deduce that

$$
\frac{\alpha}{2}\left\|u_{\alpha}\right\|_{L_{\#}^{2}\left(\mathbb{R}^{N}\right)}^{2}+\left\|\nabla u_{\alpha}\right\|_{L_{\#}^{2}\left(\mathbb{R}^{N}\right)}^{2} \leq \frac{\alpha}{2} \operatorname{meas}(P)\left|u_{0}\right|^{2}+|L|\left\|g\left(\cdot, u_{0}\right)\right\|_{L_{\#}^{2}\left(\mathbb{R}^{N}\right)}\left\|\nabla u_{\alpha}\right\|_{L_{\#}^{2}\left(\mathbb{R}^{N}\right)},
$$

and therefore

$$
\left\|\nabla u_{\alpha}\right\|_{L_{\#}^{2}\left(\mathbb{R}^{N}\right)} \leq|L|\left\|g\left(\cdot, u_{0}\right)\right\|_{L_{\#}^{2}\left(\mathbb{R}^{N}\right)}+\left(\frac{\alpha}{2} \operatorname{meas}(P)\right)^{\frac{1}{2}}\left|u_{0}\right|
$$


We consider the functions $w_{\alpha}=u_{\alpha}-\left\langle u_{\alpha}\right\rangle$, where $\left\langle u_{\alpha}\right\rangle=(\operatorname{meas}(P))^{-1} \int_{P} u_{\alpha}(x) \mathrm{d} x$. By the Poincaré inequality we deduce that $\left(w_{\alpha}\right)_{\alpha}$ is bounded in $H_{\#}^{1}\left(\mathbb{R}^{N}\right)$ and therefore there is a sequence $\left(\alpha_{k}\right)_{k}$ decreasing to 0 such that $w_{\alpha_{k}} \rightarrow w$ strongly in $L_{\#}^{2}\left(\mathbb{R}^{N}\right)$ and $w_{\alpha_{k}} \rightarrow w$ weakly in $H_{\#}^{1}\left(\mathbb{R}^{N}\right)$. We claim that the sequence $\left(\left\langle u_{\alpha_{k}}\right\rangle\right)_{k}$ is bounded. Indeed, if it is not the case there is a subsequence $\left(\alpha_{k_{l}}\right)_{l}$ decreasing to 0 such that $\lim _{l \rightarrow+\infty}\left\langle u_{l}\right\rangle=+\infty$ or $\lim _{l \rightarrow+\infty}\left\langle u_{l}\right\rangle=-\infty$, where $u_{l}:=u_{\alpha_{k_{l}}}, l \geq 1$. In the first case, by (32), (33) and (34) we have

$$
0 \leq \int_{P}\left[g\left(x, u_{l}(x)\right)-g\left(x, u_{0}\right)\right]\left[u_{l}(x)-u_{0}\right] \mathrm{d} x \leq C, \quad \forall l \geq 1 .
$$

We denote by $\left(F_{l}\right)_{l}$ the nonnegative functions

$$
F_{l}(x)=\left[g\left(x, u_{l}(x)\right)-g\left(x, u_{0}\right)\right]\left[u_{l}(x)-u_{0}\right], \quad \forall x \in \mathbb{R}^{N}, l \geq 1 .
$$

Since $\left(w_{l}\right)_{l}$ converges to $w$ in $L_{\#}^{2}\left(\mathbb{R}^{N}\right)$, we have (after extraction of a subsequence) that $\lim _{l \rightarrow+\infty} w_{l}(x)=w(x)$ a.e. $x \in \mathbb{R}^{N}$ and thus $\lim _{l \rightarrow+\infty} u_{l}(x)=+\infty$ a.e. $x \in \mathbb{R}^{N}$. Pick an arbitrary $v_{0} \in \mathbb{R}$ such that $v_{0} \geq u_{0}$ and observe that

$$
F_{l}(x) \geq\left[g\left(x, v_{0}\right)-g\left(x, u_{0}\right)\right]\left[v_{0}-u_{0}\right]=: F(x), \quad \forall l \geq l(x), \text { a.e. } x \in \mathbb{R}^{N},
$$

which implies that $\liminf _{l \rightarrow+\infty} F_{l}(x) \geq F(x)$, a.e. $x \in \mathbb{R}^{N}$. By using the Fatou lemma we deduce that for any $v_{0} \geq u_{0}$ we have

$$
0 \leq \int_{P} F(x) \mathrm{d} x \leq \int_{P} \liminf _{l \rightarrow+\infty} F_{l}(x) \mathrm{d} x \leq \liminf _{l \rightarrow+\infty} \int_{P} F_{l}(x) \mathrm{d} x \leq C
$$

We obtain $0 \leq \int_{P}\left\{g\left(x, v_{0}\right)-g\left(x, u_{0}\right)\right\} \mathrm{d} x \leq \frac{C}{v_{0}-u_{0}}, \quad \forall v_{0}>u_{0}$, or $G\left(v_{0}\right)=G\left(v_{0}\right)-G\left(u_{0}\right) \leq \frac{C}{v_{0}-u_{0}}, \quad \forall v_{0}>u_{0}$. By the hypotheses we know that there is $u_{0}^{+}>u_{0}$ such that $G\left(u_{0}^{+}\right)>0$ and thus for any $v_{0} \geq u_{0}^{+}$we have $0<G\left(u_{0}^{+}\right) \leq G\left(v_{0}\right) \leq \frac{C}{v_{0}-u_{0}}$. By letting $v_{0} \rightarrow+\infty$ we find a contradiction $0<G\left(u_{0}^{+}\right) \leq 0$. Let us analyze the case $\lim _{l \rightarrow+\infty}\left\langle u_{l}\right\rangle=-\infty$. This time we have $\lim _{l \rightarrow+\infty} u_{l}(x)=-\infty$ a.e. $x \in \mathbb{R}^{N}$ and $\liminf _{l \rightarrow+\infty} F_{l}(x) \geq F(x)$ a.e. $x \in \mathbb{R}^{N}$ and any $v_{0} \leq u_{0}$. By Fatou lemma one gets

$$
0 \leq \int_{P} F(x) \mathrm{d} x \leq \int_{P} \liminf _{l \rightarrow+\infty} F_{l}(x) \mathrm{d} x \leq \liminf _{l \rightarrow+\infty} \int_{P} F_{l}(x) \mathrm{d} x \leq C, \quad \forall v_{0} \leq u_{0} .
$$

We deduce that $0 \geq \int_{P}\left\{g\left(x, v_{0}\right)-g\left(x, u_{0}\right)\right\} \mathrm{d} x \geq \frac{C}{v_{0}-u_{0}}, \forall v_{0} \leq u_{0}$. We know that there is $u_{0}^{-}<u_{0}$ such that $G\left(u_{0}^{-}\right)<0$ and thus for any $v_{0} \leq u_{0}^{-}<u_{0}$ we have $0>G\left(u_{0}^{-}\right) \geq G\left(v_{0}\right) \geq \frac{C}{v_{0}-u_{0}}$. By letting $v_{0} \rightarrow-\infty$ we find another contradiction $0>G\left(u_{0}^{-}\right) \geq 0$. Finally we deduce that $\left(\left\langle u_{\alpha_{k}}\right\rangle\right)_{k}$ is bounded and it follows easily that $\sup _{0<\alpha \leq 1}\left|\left\langle u_{\alpha}\right\rangle\right|<+\infty$. Since we already know that $\sup _{0<\alpha \leq 1}\left\|\nabla u_{\alpha}\right\|_{L_{\#}^{2}\left(\mathbb{R}^{N}\right)}<+\infty$, we obtain by using the Poincaré inequality that $\sup _{0<\alpha \leq 1}\left\|u_{\alpha}\right\|_{H_{\#}^{1}\left(\mathbb{R}^{N}\right)}<+\infty$. We can also estimate the $L_{\#}^{2}\left(\mathbb{R}^{N}\right)$ norm of $D^{2} u_{\alpha}$ and $g\left(\cdot, u_{\alpha}(\cdot)\right)$ by performing computations similar to those in the proof of Theorem 2.1. Notice that this time the estimates are uniform w.r.t. $\alpha \in] 0,1]$ because we already have a uniform bound for the $L_{\#}^{2}\left(\mathbb{R}^{N}\right)$ norm of $u_{\alpha}$.

Proof of Theorem 1.1. Consider a sequence $\left(\alpha_{k}\right)_{k}$ converging to 0. By Proposition 2.5 we know that

$$
\sup _{k}\left\{\left\|u_{k}\right\|_{H_{\#}^{2}\left(\mathbb{R}^{N}\right)}+\left\|g\left(\cdot, u_{k}(\cdot)\right)\right\|_{L_{\#}^{2}\left(\mathbb{R}^{N}\right)}\right\}<+\infty
$$

where $\left(u_{k}\right)_{k}$ are the periodic solutions of (9) with $\alpha=\alpha_{k}, k \geq 1$. After extraction of a subsequence we can assume that there is $u \in H_{\#}^{2}\left(\mathbb{R}^{N}\right)$ such that $u_{k} \rightarrow u$ strongly in $H_{\#}^{1}\left(\mathbb{R}^{N}\right), u_{k} \rightarrow u$ weakly in $H_{\#}^{2}\left(\mathbb{R}^{N}\right), g\left(\cdot, u_{k}(\cdot)\right) \rightarrow z(\cdot)$ weakly in $L_{\#}^{2}\left(\mathbb{R}^{N}\right)$. As in the proof of Theorem 2.1 we show that $g(\cdot, u(\cdot))$ belongs to $L_{\#}^{2}\left(\mathbb{R}^{N}\right)$ and coincides with $z(\cdot)$. We find easily that $u$ solves $(2)$. From (34) we deduce also that $\|\nabla u\|_{L_{\#}^{2}\left(\mathbb{R}^{N}\right)} \leq|L|\left\|g\left(\cdot, u_{0}\right)\right\|_{L_{\#}^{2}\left(\mathbb{R}^{N}\right)}$, for any $u_{0} \in G^{-1}(0)$. 
We intend now to identify the solution obtained above as limit of penalized solutions $u=\lim _{k \rightarrow+\infty} u_{k}$. Take $v$ an arbitrary periodic solution of (2). We have

$$
\alpha_{k} u_{k}-\Delta\left(u_{k}-v\right)+g\left(x, u_{k}(x)\right)-g(x, v(x))=0, \quad x \in \mathbb{R}^{N}
$$

and therefore

$$
\alpha_{k} \int_{P} u_{k}(x)\left(u_{k}(x)-v(x)\right) \mathrm{d} x+\int_{P}\left|\nabla u_{k}-\nabla v\right|^{2} \mathrm{~d} x \leq 0
$$

which implies

$$
\int_{P}\left|u_{k}(x)\right|^{2} \mathrm{~d} x \leq \int_{P} u_{k}(x) v(x) \mathrm{d} x, \quad \forall k \geq 1
$$

After passing to the limit for $k \rightarrow+\infty$ we deduce that $u$ satisfies the following minimality property

$$
\int_{P}|u(x)|^{2} \mathrm{~d} x \leq \int_{P} u(x) v(x) \mathrm{d} x
$$

for any periodic solution of (2). In particular we have $\int_{P}|u(x)|^{2} \mathrm{~d} x \leq \int_{P}|v(x)|^{2} \mathrm{~d} x$ for any periodic solution of (2). Observe also that there is at most one solution for (2) satisfying the minimality property $(M)$. Indeed, take $u_{1}, u_{2}$ two periodic solutions of $(2)$ verifying $(M)$. We have

$$
\int_{P}\left|u_{1}(x)-u_{2}(x)\right|^{2} \mathrm{~d} x=\int_{P} u_{1}(x)\left(u_{1}(x)-u_{2}(x)\right) \mathrm{d} x+\int_{P} u_{2}(x)\left(u_{2}(x)-u_{1}(x)\right) \mathrm{d} x \leq 0,
$$

and therefore $u_{1}=u_{2}$.

Now since the minimality property $(M)$ uniquely determines the limit, we deduce that we have the global convergence $u_{\alpha} \rightarrow u$ strongly in $H_{\#}^{1}\left(\mathbb{R}^{N}\right), u_{\alpha} \rightarrow u$ weakly in $H_{\#}^{2}\left(\mathbb{R}^{N}\right), g\left(\cdot, u_{\alpha}(\cdot)\right) \rightarrow g(\cdot, u(\cdot))$ weakly in $L_{\#}^{2}\left(\mathbb{R}^{N}\right)$ for $\alpha \searrow 0$. We call the solution constructed above minimal solution.

By Sobolev inclusions $\left(H_{\#}^{1}\left(\mathbb{R}^{N}\right) \subset C_{\#}^{0}\left(\mathbb{R}^{N}\right)\right.$ if $N=1, H_{\#}^{2}\left(\mathbb{R}^{N}\right) \subset C_{\#}^{0}\left(\mathbb{R}^{N}\right)$ if $\left.N \in\{2,3\}\right)$ we know that any solution $u \in H_{\#}^{2}\left(\mathbb{R}^{N}\right), 1 \leq N \leq 3$ is bounded and therefore, the necessary conditions $\left(C_{1}\right),\left(C_{2}\right)$ are equivalent (cf. Prop. 2.1). We claim that if $1 \leq N \leq 3$, at least for nonlinear functions $g(x, u)=\beta(u)-f(x)$ we can relax the condition $\left(C_{4}\right)$ of Theorem 1.1. Actually we can prove the existence of periodic solution $u \in H_{\#}^{2}\left(\mathbb{R}^{N}\right)$ for (2) under the hypotheses $\left(H_{1}\right),\left(H_{2}\right),\left(H_{3}\right),\left(H_{4}\right)$ and condition $\left(C_{2}\right)$ which means that in these cases $\left(C_{2}\right)$ is a necessary and sufficient condition for the existence of periodic solution $u \in H_{\#}^{2}\left(\mathbb{R}^{N}\right)$. We will use the following easy lemmas.

Lemma 2.6. Assume that $v$ belongs to $W_{\#}^{1, q}\left(\mathbb{R}^{N}\right)$ for some $q>N$ and let $\langle v\rangle:=(\operatorname{meas}(P))^{-1} \int_{P} v(x) \mathrm{d} x$. 1) If meas $\{x \in P: v(x) \geq m\}>0$ then $\langle v\rangle \geq m-C\|\nabla v\|_{L_{+}^{q}\left(\mathbb{R}^{N}\right)}$, for some constant $C$ depending on $q, N$, L.

2) If meas $\{x \in P: v(x) \leq M\}>0$ then $\langle v\rangle \leq M+C\|\nabla v\|_{L_{\#}^{q}\left(\mathbb{R}^{N}\right)}$, for some constant $C$ depending on $q, N, L$.

Proof. We prove only the first statement (for the second one change $v$ to $-v$ ). Take $\left(\varphi_{k}\right)_{k} \subset C_{\#}^{1}\left(\mathbb{R}^{N}\right)$ converging towards $v$ in $W_{\#}^{1, q}\left(\mathbb{R}^{N}\right)$. After extraction of a subsequence we can suppose that $\lim _{k \rightarrow+\infty} \varphi_{k}(x)=v(x)$ a.e. $x \in \mathbb{R}^{N}$. By the hypothesis there is $x_{0} \in P$ such that $\lim _{k \rightarrow+\infty} \varphi_{k}\left(x_{0}\right)=v\left(x_{0}\right) \geq m$. Consider $P_{0}=\left\{x_{0}\right\}+P=$ $\left\{x_{0}+x: x \in P\right\}$. For any $x \in P_{0}$ we have

$$
\begin{aligned}
\varphi_{k}(x)-\varphi_{k}\left(x_{0}\right) & =\int_{0}^{1} \frac{\mathrm{d}}{\mathrm{d} \tau} \varphi_{k}\left(x_{0}+\tau\left(x-x_{0}\right)\right) \mathrm{d} \tau \\
& =\int_{0}^{1} \nabla \varphi_{k}\left(x_{0}+\tau\left(x-x_{0}\right)\right) \cdot\left(x-x_{0}\right) \mathrm{d} \tau .
\end{aligned}
$$


After integration over $P_{0}$ one gets by periodicity

$$
\begin{aligned}
\operatorname{meas}(P)\left\{\left\langle\varphi_{k}\right\rangle-\varphi_{k}\left(x_{0}\right)\right\} & =\int_{0}^{1} \int_{P_{0}} \nabla \varphi_{k}\left(x_{0}+\tau\left(x-x_{0}\right)\right) \cdot\left(x-x_{0}\right) \mathrm{d} x \mathrm{~d} \tau \\
& =\int_{0}^{1} \int_{P} \nabla \varphi_{k}\left(x_{0}+\tau y\right) \cdot y \mathrm{~d} y \mathrm{~d} \tau \\
& =: I .
\end{aligned}
$$

We can estimate $I$ as follows

$$
\begin{aligned}
|I| & \leq \int_{0}^{1}|L| \int_{P}\left|\nabla \varphi_{k}\left(x_{0}+\tau y\right)\right| \mathrm{d} y \mathrm{~d} \tau \\
& =|L| \int_{0}^{1} \frac{1}{\tau^{N}} \int_{\tau P}\left|\nabla \varphi_{k}\left(x_{0}+z\right)\right| \mathrm{d} z \mathrm{~d} \tau \\
& \leq|L| \int_{0}^{1} \frac{1}{\tau^{N}}\left(\int_{\tau P}\left|\nabla \varphi_{k}\left(x_{0}+z\right)\right|^{q} \mathrm{~d} z\right)^{\frac{1}{q}} \operatorname{meas}(P)^{\frac{1}{q^{\prime}}} \tau^{\frac{N}{q^{\prime}}} \mathrm{d} \tau \\
& \leq|L|\left\|\nabla \varphi_{k}\right\|_{L_{\#}^{q}\left(\mathbb{R}^{N}\right)} \operatorname{meas}(P)^{\frac{1}{q^{\prime}}} \int_{0}^{1} \frac{\mathrm{d} \tau}{\tau^{\frac{N}{q}}} \\
& =|L|\left\|\nabla \varphi_{k}\right\|_{L_{\#}^{q}\left(\mathbb{R}^{N}\right)} \operatorname{meas}(P)^{\frac{1}{q^{\prime}}} \frac{q}{q-N}
\end{aligned}
$$

From (35) and (36) we deduce

$$
\left\langle\varphi_{k}\right\rangle-\varphi_{k}\left(x_{0}\right) \geq-\frac{|L|}{\operatorname{meas}(P)^{\frac{1}{q}}} \frac{q}{q-N}\left\|\nabla \varphi_{k}\right\|_{L_{\#}^{q}\left(\mathbb{R}^{N}\right)}=-C(q, N, L)\left\|\nabla \varphi_{k}\right\|_{L_{\#}^{q}\left(\mathbb{R}^{N}\right)},
$$

and by letting $k \rightarrow+\infty$ one gets $\langle v\rangle \geq m-C(q, N, L)\|\nabla v\|_{L_{\#}^{q}\left(\mathbb{R}^{N}\right)}$.

Lemma 2.7. Assume that $\beta: \mathbb{R} \rightarrow \mathbb{R}$ is continuous, nondecreasing and $w$ belongs to $\operatorname{Range}(\beta)$. For any $\alpha>0$ we define $v_{\alpha}=(\alpha+\beta)^{-1}(w)$, i.e., $\alpha v_{\alpha}+\beta\left(v_{\alpha}\right)=w$. Then the sequence $\left(v_{\alpha}\right)_{\alpha}$ converges towards the element of minimal absolute value of the closed convex set $\beta^{-1}(w) \neq \emptyset$.

Proof. For any $v \in \beta^{-1}(w)$ we have $\alpha v_{\alpha}+\beta\left(v_{\alpha}\right)-\beta(v)=0, \alpha>0$, and after multiplication by $v_{\alpha}-v$ one gets $\left|v_{\alpha}\right|^{2} \leq v_{\alpha} v$. In particular we deduce that $\left(v_{\alpha}\right)_{\alpha}$ is bounded. Take now a limit point of $\left(v_{\alpha}\right)_{\alpha}$, i.e., $z=\lim _{k \rightarrow+\infty} v_{\alpha_{k}}$ with $\lim _{k \rightarrow+\infty} \alpha_{k}=0$. We deduce that $z \in \beta^{-1}(w)$ and $z(z-v) \leq 0$ for any $v \in \beta^{-1}(w)$. Therefore $z$ is the projection of 0 on the closed convex set $\beta^{-1}(w)$ and we deduce that $\left(v_{\alpha}\right)_{\alpha}$ converges as $\alpha \searrow 0$ towards $\operatorname{Proj}_{\beta^{-1}(w)}(0)$ (or to the element of minimal absolute value of the closed convex set $\beta^{-1}(w) \neq \emptyset$ ).

Proof of Theorem 1.2. Consider $\left(u_{\alpha}\right)_{\alpha}$ the sequence of periodic solutions for

$$
\alpha u_{\alpha}-\Delta u_{\alpha}+\beta\left(u_{\alpha}\right)=f(x), \quad x \in \mathbb{R}^{N} .
$$

For any $u_{0} \in \beta^{-1}(\langle f\rangle)$ we have $\alpha u_{\alpha}-\Delta u_{\alpha}+\beta\left(u_{\alpha}\right)-\beta\left(u_{0}\right)=f(x)-\langle f\rangle, \quad x \in \mathbb{R}^{N}$, and therefore we deduce as in $(33)$

$$
\begin{aligned}
\alpha \int_{P} u_{\alpha}\left(u_{\alpha}-u_{0}\right) \mathrm{d} x+\int_{P}\left|\nabla u_{\alpha}\right|^{2} \mathrm{~d} x & \leq \int_{P}(f-\langle f\rangle)\left(u_{\alpha}-u_{0}\right) \mathrm{d} x \\
& \leq|L|\|f-\langle f\rangle\|_{L_{\#}^{2}\left(\mathbb{R}^{N}\right)}\left\|\nabla u_{\alpha}\right\|_{L_{\#}^{2}\left(\mathbb{R}^{N}\right)} .
\end{aligned}
$$


By using the inequality $u_{\alpha}\left(u_{\alpha}-u_{0}\right) \geq \frac{1}{2}\left|u_{\alpha}\right|^{2}-\frac{1}{2}\left|u_{0}\right|^{2}$ we obtain

$$
\sqrt{\alpha}\left\|u_{\alpha}\right\|_{L_{\#}^{2}\left(\mathbb{R}^{N}\right)}+\left\|\nabla u_{\alpha}\right\|_{L_{\#}^{2}\left(\mathbb{R}^{N}\right)} \leq C\left(\|f-\langle f\rangle\|_{L_{\#}^{2}\left(\mathbb{R}^{N}\right)}+\sqrt{\alpha}\left|u_{0}\right|\right) .
$$

By standard computations we obtain also

$$
\begin{aligned}
\alpha \int_{P} u_{\alpha}(x)\left(\beta\left(u_{\alpha}(x)\right)-\beta\left(u_{0}\right)\right) \mathrm{d} x & +\int_{P}\left|\beta\left(u_{\alpha}(x)\right)-\beta\left(u_{0}\right)\right|^{2} \mathrm{~d} x \\
& \leq \int_{P}(f(x)-\langle f\rangle)\left(\beta\left(u_{\alpha}(x)\right)-\beta\left(u_{0}\right)\right) \mathrm{d} x
\end{aligned}
$$

and therefore by using (37) one gets

$$
\begin{aligned}
\left\|\beta\left(u_{\alpha}\right)-\beta\left(u_{0}\right)\right\|_{L_{\#}^{2}\left(\mathbb{R}^{N}\right)}^{2} & \leq \alpha\left\|u_{\alpha}\right\|_{L_{\#}^{2}\left(\mathbb{R}^{N}\right)}\left|\beta\left(u_{0}\right)\right| \operatorname{meas}(P)^{\frac{1}{2}} \\
& +\|f-\langle f\rangle\|_{L_{\#}^{2}\left(\mathbb{R}^{N}\right)}\left\|\beta\left(u_{\alpha}\right)-\beta\left(u_{0}\right)\right\|_{L_{\#}^{2}\left(\mathbb{R}^{N}\right)} \\
& \leq C \sqrt{\alpha}+\|f-\langle f\rangle\|_{L_{\#}^{2}\left(\mathbb{R}^{N}\right)}\left\|\beta\left(u_{\alpha}\right)-\beta\left(u_{0}\right)\right\|_{L_{\#}^{2}\left(\mathbb{R}^{N}\right)}, \quad \forall 0<\alpha \leq 1,
\end{aligned}
$$

which implies

$$
\left\|\beta\left(u_{\alpha}\right)-\langle f\rangle\right\|_{L_{\#}^{2}\left(\mathbb{R}^{N}\right)}=\left\|\beta\left(u_{\alpha}\right)-\beta\left(u_{0}\right)\right\|_{L_{\#}^{2}\left(\mathbb{R}^{N}\right)} \leq\|f-\langle f\rangle\|_{L_{\#}^{2}\left(\mathbb{R}^{N}\right)}+C \alpha^{\frac{1}{4}}, \quad \forall 0<\alpha \leq 1 .
$$

In order to estimate the second derivatives write as usual

$$
\alpha D_{h} u_{\alpha}-\Delta D_{h} u_{\alpha}+\beta\left(u_{\alpha}(\cdot+h)\right)-\beta\left(u_{\alpha}\right)=D_{h}(f-\langle f\rangle) .
$$

Therefore we obtain $\left\|D_{h} \nabla u_{\alpha}\right\|_{L_{\#}^{2}\left(\mathbb{R}^{N}\right)}^{2} \leq|h|\|f-\langle f\rangle\|_{L_{\#}^{2}\left(\mathbb{R}^{N}\right)}\left\|D_{h} \nabla u_{\alpha}\right\|_{L_{\#}^{2}\left(\mathbb{R}^{N}\right)}$, which implies that $\left\|D^{2} u_{\alpha}\right\|_{L_{\#}^{2}\left(\mathbb{R}^{N}\right)} \leq$ $C\|f-\langle f\rangle\|_{L_{\#}^{2}\left(\mathbb{R}^{N}\right)}, \forall \alpha>0$, for some constant not depending on $\alpha>0$. Notice that we have

$$
\alpha \int_{P} u_{\alpha}(x) \mathrm{d} x+\int_{P} \beta\left(u_{\alpha}(x)\right) \mathrm{d} x=\int_{P} f(x) \mathrm{d} x .
$$

Denote by $\left(v_{\alpha}\right)_{\alpha}$ the sequence given by

$$
v_{\alpha}=(\alpha+\beta)^{-1}(\langle f\rangle), \quad \forall \alpha>0 .
$$

By Lemma 2.7 we know that $\left(v_{\alpha}\right)_{\alpha}$ converges to the element of minimal absolute value of the closed convex set $\beta^{-1}(\langle f\rangle) \neq \emptyset, \lim _{\alpha \searrow 0} v_{\alpha}=\tilde{u}_{0}=\operatorname{Proj}_{\beta^{-1}(\langle f\rangle)}(0)$. The equation (39) can be written

$$
\alpha \int_{P} v_{\alpha} \mathrm{d} x+\int_{P} \beta\left(v_{\alpha}\right) \mathrm{d} x=\int_{P} f(x) \mathrm{d} x, \quad \forall \alpha>0 .
$$

Combining (38) and (40) yields $\int_{P}\left\{\alpha\left(u_{\alpha}(x)-v_{\alpha}\right)+\beta\left(u_{\alpha}(x)\right)-\beta\left(v_{\alpha}\right)\right\} \mathrm{d} x=0, \quad \forall \alpha>0$. We deduce that there are measurable sets $A_{\alpha}^{ \pm}$, meas $\left(A_{\alpha}^{ \pm}\right)>0$ such that $u_{\alpha}(x)-v_{\alpha} \geq 0$ a.e. $x \in A_{\alpha}^{-}$and $u_{\alpha}(x)-v_{\alpha} \leq 0$ a.e. $x \in A_{\alpha}^{+}$. If $N=1$ the hypotheses of Lemma 2.6 are verified with $q=2$. In the cases $N \in\{2,3\}$, since $\left\|\nabla u_{\alpha}\right\|_{H_{\#}^{1}\left(\mathbb{R}^{N}\right)} \leq C\left(\|f-\langle f\rangle\|_{L_{\#}^{2}\left(\mathbb{R}^{N}\right)}+\sqrt{\alpha}\left|u_{0}\right|\right)$ where $C$ does not depend on $\alpha$ we deduce by Sobolev inclusions that $\left\|\nabla u_{\alpha}\right\|_{L_{\#}^{q}\left(\mathbb{R}^{N}\right)} \leq C\left(\|f-\langle f\rangle\|_{L_{\#}^{2}\left(\mathbb{R}^{N}\right)}+\sqrt{\alpha}\left|u_{0}\right|\right), \quad \forall 0<\alpha \leq 1$, for some $q>N$. By Lemma 2.6 we deduce that for any $0<\alpha \leq 1$ we have

$$
\left|\left\langle u_{\alpha}\right\rangle-v_{\alpha}\right| \leq C\left(\|f-\langle f\rangle\|_{L_{\#}^{2}\left(\mathbb{R}^{N}\right)}+\sqrt{\alpha}\left|u_{0}\right|\right),
$$


and therefore $\left(\left\langle u_{\alpha}\right\rangle\right)_{\alpha}$ is bounded. We conclude as in the proof of Theorem 1.1 that $\left(u_{\alpha}\right)_{\alpha}$ converges to a periodic solution $u$ of $(5)$. Moreover, the solution satisfies

$$
\left|\langle u\rangle-\tilde{u}_{0}\right|+\left\|u-\tilde{u}_{0}\right\|_{H_{\#}^{2}\left(\mathbb{R}^{N}\right)}+\|\beta(u)-\langle f\rangle\|_{L_{\#}^{2}\left(\mathbb{R}^{N}\right)} \leq C\|f-\langle f\rangle\|_{L_{\#}^{2}\left(\mathbb{R}^{N}\right)} .
$$

\subsection{Stability}

We analyze now the stability of the minimal solution of (5) w.r.t. $f$.

Theorem 2.2. Assume that $\beta: \mathbb{R} \rightarrow \mathbb{R}$ is continuous, nondecreasing, $\beta(0)=0$ and $f_{1}, f_{2}: \mathbb{R}^{N} \rightarrow \mathbb{R} \in L_{\#}^{2}\left(\mathbb{R}^{N}\right)$ satisfy $\left\langle f_{1}\right\rangle=\left\langle f_{2}\right\rangle \in \operatorname{Range}(\beta)$. Denote by $\left(u_{k}\right)_{1 \leq k \leq 2}$ the minimal periodic solutions of $-\Delta u+\beta(u)=f_{k}(x)$, $x \in \mathbb{R}^{N}, 1 \leq k \leq 2$. Then we have the inequality $\left\|\nabla u_{1}-\nabla u_{2}\right\|_{L_{\#}^{2}\left(\mathbb{R}^{N}\right)} \leq|L|\left\|f_{1}-f_{2}\right\|_{L_{\#}^{2}\left(\mathbb{R}^{N}\right)}, \forall N \geq 1$. If $N=1$ we have also

$$
\left|\left\langle u_{1}\right\rangle-\left\langle u_{2}\right\rangle\right| \leq 2 L_{1}^{\frac{3}{2}}\left\|f_{1}-f_{2}\right\|_{L_{\#}^{2}(\mathbb{R})}, \quad\left\|u_{1}-u_{2}\right\|_{L_{\#}^{2}(\mathbb{R})} \leq 3 L_{1}^{2}\left\|f_{1}-f_{2}\right\|_{L_{\#}^{2}(\mathbb{R})} .
$$

Proof. By the construction of the minimal periodic solution we have $u_{k}=\lim _{\alpha \searrow 0} u_{k, \alpha}$ in $H_{\#}^{1}\left(\mathbb{R}^{N}\right)$ where $u_{k, \alpha}$ solves $\alpha u_{k, \alpha}-\Delta u_{k, \alpha}+\beta\left(u_{k, \alpha}\right)=f_{k}, \quad x \in \mathbb{R}^{N}, \alpha>0,1 \leq k \leq 2$. Therefore we have $\alpha\left(u_{1, \alpha}-u_{2, \alpha}\right)-\Delta\left(u_{1, \alpha}-\right.$ $\left.u_{2, \alpha}\right)+\beta\left(u_{1, \alpha}\right)-\beta\left(u_{2, \alpha}\right)=f_{1}-f_{2}$, which implies as in the proof of Proposition 2.5 (see (33)) that

$$
\begin{aligned}
\int_{P}\left|\nabla u_{1, \alpha}-\nabla u_{2, \alpha}\right|^{2} \mathrm{~d} x & \leq \int_{P}\left(f_{1}-f_{2}\right)\left(u_{1, \alpha}-u_{2, \alpha}\right) \mathrm{d} x \\
& \leq|L|\left\|f_{1}-f_{2}\right\|_{L_{\#}^{2}\left(\mathbb{R}^{N}\right)}\left\|\nabla u_{1, \alpha}-\nabla u_{2, \alpha}\right\|_{L_{\#}^{2}\left(\mathbb{R}^{N}\right)} .
\end{aligned}
$$

Therefore one gets

$$
\left\|\nabla u_{1, \alpha}-\nabla u_{2, \alpha}\right\|_{L_{\#}^{2}\left(\mathbb{R}^{N}\right)} \leq|L|\left\|f_{1}-f_{2}\right\|_{L_{\#}^{2}\left(\mathbb{R}^{N}\right)}, \forall \alpha>0
$$

and the conclusion follows by letting $\alpha \searrow 0$. We have also

$$
\int_{P}\left\{\alpha\left(u_{1, \alpha}(x)-u_{2, \alpha}(x)\right)+\beta\left(u_{1, \alpha}(x)\right)-\beta\left(u_{2, \alpha}(x)\right)\right\} \mathrm{d} x=0,
$$

and thus there are measurable sets $A_{\alpha}^{ \pm}$, meas $\left(A_{\alpha}^{ \pm}\right)>0$ such that $u_{1, \alpha}(x)-u_{2, \alpha}(x) \geq 0$ a.e. $x \in A_{\alpha}^{+}$and $u_{1, \alpha}(x)-u_{2, \alpha}(x) \leq 0$ a.e. $x \in A_{\alpha}^{-}$. By Lemma 2.6 we deduce that if $N=1$ we have

$$
\left|\left\langle u_{1, \alpha}\right\rangle-\left\langle u_{2, \alpha}\right\rangle\right| \leq 2 L_{1}^{\frac{1}{2}}\left\|\nabla u_{1, \alpha}-\nabla u_{2, \alpha}\right\|_{L_{\#}^{2}(\mathbb{R})} \leq 2 L_{1}^{\frac{3}{2}}\left\|f_{1}-f_{2}\right\|_{L_{\#}^{2}(\mathbb{R})}, \alpha>0 .
$$

We deduce by the Poincaré inequality that

$$
\begin{aligned}
\left\|u_{1, \alpha}-u_{2, \alpha}\right\|_{L_{\#}^{2}(\mathbb{R})} & \leq L_{1}^{\frac{1}{2}}\left|\left\langle u_{1, \alpha}\right\rangle-\left\langle u_{2, \alpha}\right\rangle\right|+\left\|u_{1, \alpha}-u_{2, \alpha}-\left\langle u_{1, \alpha}-u_{2, \alpha}\right\rangle\right\|_{L_{\#}^{2}(\mathbb{R})} \\
& \leq 2 L_{1}^{2}\left\|f_{1}-f_{2}\right\|_{L_{\#}^{2}(\mathbb{R})}+L_{1}\left\|\nabla u_{1, \alpha}-\nabla u_{2, \alpha}\right\|_{L_{\#}^{2}(\mathbb{R})} \\
& \leq 3 L_{1}^{2}\left\|f_{1}-f_{2}\right\|_{L_{\#}^{2}(\mathbb{R})}, \alpha>0 .
\end{aligned}
$$

By letting $\alpha \searrow 0$ we obtain that $\left\|u_{1}-u_{2}\right\|_{L_{\#}^{2}(\mathbb{R})} \leq 3 L_{1}^{2}\left\|f_{1}-f_{2}\right\|_{L_{\#}^{2}(\mathbb{R})}$.

\subsection{Homogenization}

Consider $f: \mathbb{R}^{N} \rightarrow \mathbb{R}$ a $L$ periodic function such that $\langle f\rangle \in \operatorname{Range}(\beta)$. We denote by $\left(f_{n}\right)_{n}$ the $\frac{L}{n}$ periodic functions given by $f_{n}(x)=f(n x)$. Since $\left\langle f_{n}\right\rangle=\langle f\rangle \in \operatorname{Range}(\beta)$, for any $n \geq 1$ we can solve $-\Delta u_{n}+\beta\left(u_{n}\right)=f_{n}$ in $\mathbb{R}^{N}$ and the natural question is what happens with the sequence of solutions $\left(u_{n}\right)_{n}$ for large $n$. The answer is given by the following theorem. 
Theorem 2.3. Assume that $\beta: \mathbb{R} \rightarrow \mathbb{R}$ is continuous, nondecreasing, $\beta(0)=0, f: \mathbb{R}^{N} \rightarrow \mathbb{R}$ belongs to $L_{\#}^{2}\left(\mathbb{R}^{N}\right),\langle f\rangle \in \operatorname{Range}(\beta)$. For any $n \geq 1$ we consider the $\frac{L}{n}$ periodic function given by $f_{n}(x)=f(n x), x \in \mathbb{R}^{N}$ and we denote by $u_{n}$ the minimal periodic solution of $-\Delta u+\beta(u)=f_{n}(x)$ in $\mathbb{R}^{N}$. Then we have the inequality

$$
\left\|\nabla u_{n}\right\|_{L_{\#}^{2}\left(\mathbb{R}^{N}\right)} \leq \frac{|L|}{n}\|f-\langle f\rangle\|_{L_{\#}^{2}\left(\mathbb{R}^{N}\right)}, \quad \forall n \geq 1, \forall N \geq 1 .
$$

Moreover, if $N=1$ then there is a constant $C_{1}$ such that $\left\|u_{n}-\tilde{u}_{0}\right\|_{L_{\#}^{2}(\mathbb{R})} \leq \frac{C_{1}}{n}\|f-\langle f\rangle\|_{L_{\#}^{2}(\mathbb{R})}, n \geq 1$, if $N=2$ then for any $\theta \in] 0,1\left[\right.$ there is a constant $C_{2}(\theta)$ such that $\left\|u_{n}-\tilde{u}_{0}\right\|_{L_{\#}^{2}\left(\mathbb{R}^{2}\right)} \leq \frac{C_{2}(\theta)}{n^{\theta}}\|f-\langle f\rangle\|_{L_{\#}^{2}\left(\mathbb{R}^{2}\right)}, n \geq 1$ and if $N=3$ then for any $\theta \in] 0, \frac{1}{2}\left[\right.$ there is a constant $C_{3}(\theta)$ such that $\left\|u_{n}-\tilde{u}_{0}\right\|_{L_{\#}^{2}\left(\mathbb{R}^{3}\right)} \leq \frac{C_{3}(\theta)}{n^{\theta}}\|f-\langle f\rangle\|_{L_{\#}^{2}\left(\mathbb{R}^{3}\right)}$, $n \geq 1$, where $\tilde{u}_{0}$ is the element of minimal absolute value of the closed convex set $\beta^{-1}(\langle f\rangle) \neq \emptyset$.

Proof. We have $\nabla u_{n}=\lim _{\alpha \searrow 0} \nabla u_{n, \alpha}$ in $L_{\#}^{2}\left(\mathbb{R}^{N}\right), \tilde{u}_{0}=\lim _{\alpha \searrow 0} v_{\alpha}$ in $\mathbb{R}$ where

$$
\begin{gathered}
\alpha u_{n, \alpha}(x)-\Delta u_{n, \alpha}+\beta\left(u_{n, \alpha}(x)\right)=f_{n}(x), \quad x \in \mathbb{R}^{N}, \alpha>0, n \geq 1, \\
\alpha v_{\alpha}+\beta\left(v_{\alpha}\right)=\langle f\rangle, \alpha>0 .
\end{gathered}
$$

We introduce the $L$ periodic functions $v_{n, \alpha}(x)=u_{n, \alpha}\left(\frac{x}{n}\right), x \in \mathbb{R}^{N}, n \geq 1, \alpha>0$. Observe that $v_{n, \alpha}, v_{\alpha}$ satisfy

$$
\frac{\alpha}{n^{2}} v_{n, \alpha}(x)-\Delta v_{n, \alpha}+\frac{\beta\left(v_{n, \alpha}(x)\right)}{n^{2}}=\frac{f(x)}{n^{2}}, x \in \mathbb{R}^{N}, \frac{\alpha}{n^{2}} v_{\alpha}-\Delta v_{\alpha}+\frac{\beta\left(v_{\alpha}\right)}{n^{2}}=\frac{\langle f\rangle}{n^{2}} .
$$

By Theorem 2.2 we obtain

$$
\frac{1}{n}\left\|\nabla u_{n, \alpha}\right\|_{L_{\#}^{2}\left(\mathbb{R}^{N}\right)}=\left\|\nabla v_{n, \alpha}\right\|_{L_{\#}^{2}\left(\mathbb{R}^{N}\right)} \leq \frac{|L|}{n^{2}}\|f-\langle f\rangle\|_{L_{\#}^{2}\left(\mathbb{R}^{N}\right)}, n \geq 1, \alpha>0,
$$

and by letting $\alpha \searrow 0$ one gets $\left\|\nabla u_{n}\right\|_{L_{\#}^{2}\left(\mathbb{R}^{N}\right)} \leq \frac{|L|}{n}\|f-\langle f\rangle\|_{L_{\#}^{2}\left(\mathbb{R}^{N}\right)}, n \geq 1$. We have

$$
\frac{\alpha}{n^{2}}\left(v_{n, \alpha}(x)-v_{\alpha}\right)-\Delta\left(v_{n, \alpha}-v_{\alpha}\right)+\frac{1}{n^{2}}\left(\beta\left(v_{n, \alpha}(x)\right)-\beta\left(v_{\alpha}\right)\right)=\frac{1}{n^{2}}(f-\langle f\rangle),
$$

and as usual we obtain $\left\|D^{2} v_{n, \alpha}\right\|_{L_{\#}^{2}\left(\mathbb{R}^{N}\right)} \leq \frac{C}{n^{2}}\|f-\langle f\rangle\|_{L_{\#}^{2}\left(\mathbb{R}^{N}\right)}$, which implies that $\left\|D^{2} u_{n, \alpha}\right\|_{L_{\#}^{2}\left(\mathbb{R}^{N}\right)} \leq C \| f-$ $\langle f\rangle \|_{L_{\#}^{2}\left(\mathbb{R}^{N}\right)}$. Since

$$
\int_{P}\left\{\alpha\left(u_{n, \alpha}(x)-v_{\alpha}\right)+\beta\left(u_{n, \alpha}(x)\right)-\beta\left(v_{\alpha}\right)\right\} \mathrm{d} x=0,
$$

we deduce that there are measurable sets $A_{n, \alpha}^{ \pm}, \operatorname{meas}\left(A_{n, \alpha}^{ \pm}\right)>0$ such that $\left(u_{n, \alpha}-v_{\alpha}\right) \mathbf{1}_{A_{n, \alpha}^{+}} \geq 0,\left(u_{n, \alpha}-\right.$ $\left.v_{\alpha}\right) \mathbf{1}_{A_{n, \alpha}^{-}} \leq 0$. By applying Lemma 2.6 we deduce in the case $N=1$

$$
\left|\left\langle u_{n, \alpha}\right\rangle-v_{\alpha}\right| \leq 2 L_{1}^{\frac{1}{2}}\left\|\nabla u_{n, \alpha}\right\|_{L_{\#}^{2}(\mathbb{R})} \leq \frac{2}{n} L_{1}^{\frac{3}{2}}\|f-\langle f\rangle\|_{L_{\#}^{2}(\mathbb{R})}, n \geq 1, \alpha>0,
$$

which implies

$$
\left|\left\langle u_{n}\right\rangle-\tilde{u}_{0}\right| \leq \frac{2}{n} L_{1}^{\frac{3}{2}}\|f-\langle f\rangle\|_{L_{\#}^{2}(\mathbb{R})}, n \geq 1 .
$$

By using Poincaré inequality one gets

$$
\left\|u_{n}-\tilde{u}_{0}\right\|_{L_{\#}^{2}(\mathbb{R})} \leq \frac{3 L_{1}^{2}}{n}\|f-\langle f\rangle\|_{L_{\#}^{2}(\mathbb{R})} .
$$


Suppose now that $N \in\{2,3\}$. We deduce that $\left\|\nabla u_{n, \alpha}\right\|_{H_{\#}^{1}\left(\mathbb{R}^{N}\right)} \leq C\|f-\langle f\rangle\|_{L_{\#}^{2}\left(\mathbb{R}^{N}\right)}$ and by Sobolev inclusions we have $\left\|\nabla u_{n, \alpha}\right\|_{L_{\#}^{q}\left(\mathbb{R}^{N}\right)} \leq C(q)\|f-\langle f\rangle\|_{L_{\#}^{2}\left(\mathbb{R}^{N}\right)}$ for $1 \leq q<+\infty$ if $N=2$ and $1 \leq q \leq 6$ if $N=3$. If $N=2$ take $\theta \in] 0,1[, q, r$ such that $2<q<r$ and $1 / q-1 / r=\theta(1 / 2-1 / r)$. By using Sobolev and interpolation inequalities one gets

$$
\begin{aligned}
\left\|\nabla u_{n, \alpha}\right\|_{L_{\#}^{q}\left(\mathbb{R}^{2}\right)} & \leq\left\|\nabla u_{n, \alpha}\right\|_{L_{\#}^{2}\left(\mathbb{R}^{2}\right)}^{\theta}\left\|\nabla u_{n, \alpha}\right\|_{L_{\#}^{r}\left(\mathbb{R}^{2}\right)}^{1-\theta} \\
& \leq\left\|\nabla u_{n, \alpha}\right\|_{L_{\#}^{2}\left(\mathbb{R}^{2}\right)} C(r)\left\|\nabla u_{n, \alpha}\right\|_{H_{\#}^{1}-\theta}^{\left.1 \mathbb{R}^{2}\right)} \\
& \leq C(r)\left\|\nabla u_{n, \alpha}\right\|_{L_{\#}^{2}\left(\mathbb{R}^{2}\right)}^{\theta}\left(\left\|\nabla u_{n, \alpha}\right\|_{L_{\#}^{2}-\left(\mathbb{R}^{2}\right)}^{1}+\left\|D^{2} u_{n, \alpha}\right\|_{L_{\#}^{2}\left(\mathbb{R}^{2}\right)}^{1-\theta}\right) \\
& \left.\leq C(r)\left\|\nabla u_{n, \alpha}\right\|_{L_{\#}^{2}\left(\mathbb{R}^{2}\right)}+C(r, q)\left\|\nabla u_{n, \alpha}\right\|_{L_{\#}^{2}\left(\mathbb{R}^{2}\right)}^{\theta}\|f-\langle f\rangle\|_{L_{\#}^{2}-\theta}^{1-\theta} \mathbb{R}^{2}\right) \\
& \leq \frac{C(\theta)}{n^{\theta}}\|f-\langle f\rangle\|_{L_{\#}^{2}\left(\mathbb{R}^{2}\right)} .
\end{aligned}
$$

By using Lemma 2.6 one gets

$$
\left|\left\langle u_{n, \alpha}\right\rangle-v_{\alpha}\right| \leq C(q)\left\|\nabla u_{n, \alpha}\right\|_{L_{\#}^{q}\left(\mathbb{R}^{2}\right)} \leq \frac{C(\theta)}{n^{\theta}}\|f-\langle f\rangle\|_{L_{\#}^{2}\left(\mathbb{R}^{2}\right)}, \alpha>0, n \geq 1,
$$

and therefore by letting $\alpha \searrow 0$ we deduce that

$$
\left|\left\langle u_{n}\right\rangle-\tilde{u}_{0}\right| \leq \frac{C(\theta)}{n^{\theta}}\|f-\langle f\rangle\|_{L_{\#}^{2}\left(\mathbb{R}^{2}\right)}, n \geq 1 .
$$

Finally we obtain by using the Poincaré inequality

$$
\left\|u_{n}-\tilde{u}_{0}\right\|_{L_{\#}^{2}\left(\mathbb{R}^{2}\right)} \leq\left\|u_{n}-\left\langle u_{n}\right\rangle\right\|_{L_{\#}^{2}\left(\mathbb{R}^{2}\right)}+\left\|\left\langle u_{n}\right\rangle-\tilde{u}_{0}\right\|_{L_{\#}^{2}\left(\mathbb{R}^{2}\right)} \leq \frac{C(\theta)}{n^{\theta}}\|f-\langle f\rangle\|_{L_{\#}^{2}\left(\mathbb{R}^{2}\right)} .
$$

In the case $N=3$ observe that for any $\theta \in] 0,1 / 2[$ there is $q \in] 3,6[$ such that $\theta=3(1 / q-1 / 6)$. As before we have

$$
\begin{aligned}
\left\|\nabla u_{n, \alpha}\right\|_{L_{\#}^{q}\left(\mathbb{R}^{3}\right)} & \leq\left\|\nabla u_{n, \alpha}\right\|_{L_{\#}^{2}\left(\mathbb{R}^{3}\right)}^{\theta}\left\|\nabla u_{n, \alpha}\right\|_{L_{\#}^{6}}^{1-\theta} \\
& \leq C\left\|\nabla u_{n, \alpha}\right\|_{L_{\#}^{2}\left(\mathbb{R}^{3}\right)}^{\theta}\left(\left\|\nabla u_{n, \alpha}\right\|_{L_{\#}^{2}\left(\mathbb{R}^{3}\right)}^{1-\theta}+\left\|D^{2} u_{n, \alpha}\right\|_{L_{\#}^{2}\left(\mathbb{R}^{3}\right)}^{1-\theta}\right) \\
& \leq \frac{C(\theta)}{n^{\theta}}\|f-\langle f\rangle\|_{L_{\#}^{2}\left(\mathbb{R}^{3}\right)}, \alpha>0, n \geq 1,
\end{aligned}
$$

and similarly we deduce that

$$
\left\|u_{n}-\tilde{u}_{0}\right\|_{L_{\#}^{2}\left(\mathbb{R}^{3}\right)} \leq \frac{C(\theta)}{n^{\theta}}\|f-\langle f\rangle\|_{L_{\#}^{2}\left(\mathbb{R}^{3}\right)}, n \geq 1 .
$$

\section{EXISTENCE OF PERIODIC SOLUTION FOR NONINCREASING NONLINEARITIES}

In this section we consider nonlinear elliptic equations in one dimension

$$
-u^{\prime \prime}(x)+g_{1}(x, u(x))=0, \quad x \in \mathbb{R},
$$

where $g_{1}: \mathbb{R} \times \mathbb{R} \rightarrow \mathbb{R}$ is nonincreasing w.r.t. $u$. It is convenient to represent the function $g_{1}$ as

$$
g_{1}(x, u)=-g(x, u)-f(x), \quad(x, u) \in \mathbb{R} \times \mathbb{R},
$$


where $g(x, u)=-g_{1}(x, u)+g_{1}(x, 0), \forall(x, u) \in \mathbb{R} \times \mathbb{R}$ and $f(x)=-g_{1}(x, 0), \forall x \in \mathbb{R}$. The above equation becomes

$$
-u^{\prime \prime}(x)-g(x, u(x))=f(x), \quad x \in \mathbb{R} .
$$

We suppose that the functions $f, g$ verify the hypotheses

$$
\begin{aligned}
& \left(H_{1}\right) \quad g(x, \cdot) \text { is continuous and nondecreasing a.e. } x \in \mathbb{R} ; \\
& \left(H_{2}\right) \quad f, g \text { are } L_{1} \text { periodic w.r.t. } x ; \\
& \left(H_{3}^{\prime}\right) \quad \exists K>0 \text { such that } g(x, u)-g(x, v) \leq K(u-v), \text { a.e. } x \in \mathbb{R}, \forall u \geq v ; \\
& \left(H_{4}^{\prime}\right) \quad f \in L_{\#}^{2}(\mathbb{R}) ; \\
& \left(H_{5}^{\prime}\right) \quad g(\cdot, 0)=0 .
\end{aligned}
$$

Observe that $|g(x, u)| \leq K|u|$ and thus $g(\cdot, u) \in L_{\#}^{1}(\mathbb{R}) \cap L_{\#}^{2}(\mathbb{R})$ for any $u \in \mathbb{R}$. Therefore the function $G(u)=$ $\int_{P} g(x, u) \mathrm{d} x$ is well defined for any $u \in \mathbb{R}$. Moreover if $u \in L_{\#}^{1}(\mathbb{R}) \cap L_{\#}^{2}(\mathbb{R})$ then $g(\cdot, u(\cdot)) \in L_{\#}^{1}(\mathbb{R}) \cap L_{\#}^{2}(\mathbb{R})$.

Definition 3.1. We say that $u \in H_{\#}^{1}(\mathbb{R})$ is a periodic solution for (42) iff

$$
\int_{P} u^{\prime}(x) v^{\prime}(x) \mathrm{d} x-\int_{P} g(x, u(x)) v(x) \mathrm{d} x=\int_{P} f(x) v(x) \mathrm{d} x, \quad \forall v \in H_{\#}^{1}(\mathbb{R}) .
$$

Obviously, if there is a periodic solution $u \in H_{\#}^{1}(\mathbb{R})$ for $(42)$, then

$$
\left(C_{1}^{\prime}\right) \quad \int_{P} g(x, u(x)) \mathrm{d} x+\int_{P} f(x) \mathrm{d} x=0 .
$$

As before, since $u$ is bounded, the above condition is equivalent to

$$
\left(C_{2}^{\prime}\right) \quad \exists u_{0} \in \mathbb{R}: \int_{P} g\left(x, u_{0}\right) \mathrm{d} x+\int_{P} f(x) \mathrm{d} x=0 .
$$

We intend to prove that under the condition $\left(C_{2}^{\prime}\right)$ there are periodic solutions for (42) provided that $K$ is small enough. We will use the following existence and uniqueness result for nondecreasing Lipschitz nonlinearities.

Proposition 3.1. Assume that the functions $f, g$ satisfy the hypotheses $\left(H_{1}\right),\left(H_{2}\right),\left(H_{3}^{\prime}\right),\left(H_{4}^{\prime}\right),\left(H_{5}^{\prime}\right)$. Then for any $\alpha>0$ there is a unique periodic solution for

$$
\alpha u(x)-u^{\prime \prime}(x)+g(x, u(x))=f(x), \quad x \in \mathbb{R} .
$$

Proof. By using the same method as in the proof of Theorem 2.1 we show that for any $\varepsilon>0$ there is a periodic solution $u_{\varepsilon}$ for

$$
\alpha u_{\varepsilon}(x)-u_{\varepsilon}^{\prime \prime}(x)+g_{\varepsilon}\left(x, u_{\varepsilon}(x)\right)=f(x), \quad x \in \mathbb{R},
$$

where $g_{\varepsilon}(x, u)=g\left(x,(1+\varepsilon g(x, \cdot))^{-1}(u)\right),(x, u) \in \mathbb{R} \times \mathbb{R}$. We deduce as usual that $\left\|u_{\varepsilon}\right\|_{H_{\#}^{1}(\mathbb{R})} \leq\|f\|_{L_{\#}^{2}(\mathbb{R})} / \min \{1, \alpha\}$, for any $\varepsilon>0$ and the estimate of the $L_{\#}^{2}(\mathbb{R})$ norm of $u_{\varepsilon}^{\prime \prime}$ follows immediately since in this case $\left|g_{\varepsilon}\left(x, u_{\varepsilon}(x)\right)\right| \leq$ $K\left|u_{\varepsilon}(x)\right|, x \in \mathbb{R}, \varepsilon>0$. Actually we have $\left|g_{\varepsilon}\left(x, v_{1}\right)-g_{\varepsilon}\left(x, v_{2}\right)\right| \leq K\left|v_{1}-v_{2}\right|$ for any $\left(x, v_{1}, v_{2}\right) \in \mathbb{R}^{3}, \varepsilon>0$ and we can pass easily to the limit for $\varepsilon \searrow 0$ in (44). 
Theorem 3.1. Assume that $f, g$ satisfy the hypotheses $\left(H_{1}\right),\left(H_{2}\right),\left(H_{3}^{\prime}\right),\left(H_{4}^{\prime}\right),\left(H_{5}^{\prime}\right)$. If $-\int_{P} f(x) \mathrm{d} x \in \operatorname{Range}(G)$ and $K$ is small enough, i.e., $K L_{1}^{2}<2$, then there is at least one periodic solution u for (42) satisfying

$$
\begin{gathered}
\left\|u-\tilde{u}_{0}\right\|_{L_{\#}^{2}(\mathbb{R})} \leq \frac{\sqrt{2} L_{1}^{2}}{2-L_{1}^{2} K}\left\|g\left(\cdot, \tilde{u}_{0}\right)+f\right\|_{L_{\#}^{2}(\mathbb{R})}, \\
\left\|u-\tilde{u}_{0}\right\|_{L^{\infty}(\mathbb{R})} \leq \frac{2 L_{1}^{\frac{3}{2}}}{2-L_{1}^{2} K}\left\|g\left(\cdot, \tilde{u}_{0}\right)+f\right\|_{L_{\#}^{2}(\mathbb{R})}, \\
\left\|u^{\prime}\right\|_{L_{\#}^{2}(\mathbb{R})} \leq \frac{2 L_{1}}{2-L_{1}^{2} K}\left\|g\left(\cdot, \tilde{u}_{0}\right)+f\right\|_{L_{\#}^{2}(\mathbb{R})},
\end{gathered}
$$

where $\tilde{u}_{0}$ is the element of minimal absolute value of the closed convex set $G^{-1}\left(-\int_{P} f(x) \mathrm{d} x\right)$. If the function $g$ is strictly increasing w.r.t. $u$ a.e. $x \in \mathbb{R}$ then the solution is unique.

Proof. For any $\alpha>0$ we define the application $\tilde{T}_{\alpha}: L_{\#}^{2}(\mathbb{R}) \rightarrow L_{\#}^{2}(\mathbb{R}), \tilde{T}_{\alpha} v=u$ where $u$ is the unique periodic solution (cf. Prop. 3.1) for

$$
\alpha^{2} u(x)-u^{\prime \prime}(x)+\alpha g(x, u(x))=\alpha(1+\alpha) v(x)+(1+\alpha) g(x, v(x))+f(x), x \in \mathbb{R} .
$$

Since $-\int_{P} f(x) \mathrm{d} x \in \operatorname{Range}(G)$ the sequence $\left(v_{\alpha}\right)_{\alpha}$ given by

$$
\alpha \operatorname{meas}(P) v_{\alpha}+G\left(v_{\alpha}\right)+\int_{P} f(x) \mathrm{d} x=0, \alpha>0,
$$

converges to $\tilde{u}_{0}=\operatorname{Proj}_{G^{-1}\left(-\int_{P} f(x) \mathrm{d} x\right)}(0)$ (cf. Lem. 2.7). We consider $T_{\alpha}$ the restriction of $\tilde{T}_{\alpha}$ to $X_{\alpha}=H_{\#}^{1}(\mathbb{R}) \cap$ $\left\{v \in L_{\#}^{2}(\mathbb{R}): \alpha \int_{P} v(x) \mathrm{d} x+\int_{P} g(x, v(x)) \mathrm{d} x+\int_{P} f(x) \mathrm{d} x=0\right\}$. Observe that $X_{\alpha}$ is nonempty since $v_{\alpha} \in X_{\alpha}$ and also that $T_{\alpha}\left(X_{\alpha}\right) \subset X_{\alpha}$. We denote by $d(\cdot, \cdot)$ the application $d: X_{\alpha} \times X_{\alpha} \rightarrow \mathbb{R}, d\left(v_{1}, v_{2}\right)=\left\|v_{1}^{\prime}-v_{2}^{\prime}\right\|_{L_{\#}^{2}(\mathbb{R})}$ for any $v_{1}, v_{2} \in X_{\alpha}$. We claim that $d$ is a metric on $X_{\alpha}$ equivalent with the metric induced by the norm of $H_{\#}^{1}(\mathbb{R})$. Indeed, if $v_{1}, v_{2} \in X_{\alpha}$ then

$$
\alpha \int_{P}\left(v_{1}(x)-v_{2}(x)\right) \mathrm{d} x+\int_{P}\left(g\left(x, v_{1}(x)\right)-g\left(x, v_{2}(x)\right)\right) \mathrm{d} x=0,
$$

and since $v_{1}, v_{2}$ are continuous there is $x_{\alpha}$ such that $\alpha\left(v_{1}\left(x_{\alpha}\right)-v_{2}\left(x_{\alpha}\right)\right)+g\left(x_{\alpha}, v_{1}\left(x_{\alpha}\right)\right)-g\left(x_{\alpha}, v_{2}\left(x_{\alpha}\right)\right)=0$, which implies that $v_{1}\left(x_{\alpha}\right)=v_{2}\left(x_{\alpha}\right)$ as $g$ is nondecreasing w.r.t. $u$. Integrating $v_{1}^{\prime}-v_{2}^{\prime}$ between $x_{\alpha}$ and $x$ we deduce that

$$
\left\|v_{1}-v_{2}\right\|_{L_{\#}^{2}(\mathbb{R})} \leq \frac{L_{1}}{\sqrt{2}}\left\|v_{1}^{\prime}-v_{2}^{\prime}\right\|_{L_{\#}^{2}(\mathbb{R})}, \quad\left\|v_{1}-v_{2}\right\|_{L^{\infty}(\mathbb{R})} \leq L_{1}^{\frac{1}{2}}\left\|v_{1}^{\prime}-v_{2}^{\prime}\right\|_{L_{\#}^{2}(\mathbb{R})},
$$

and

$$
\left\|v_{1}-v_{2}\right\|_{H_{\#}^{1}(\mathbb{R})} \leq\left(\frac{L_{1}^{2}}{2}+1\right)^{\frac{1}{2}} d\left(v_{1}, v_{2}\right) .
$$

Note also that $\left(X_{\alpha}, d\right)$ is a complete metric space: if $\left(v_{n}\right)_{n} \subset X_{\alpha}$ is a sequence such that $\lim _{m, n \rightarrow+\infty} d\left(v_{n}, v_{m}\right)=$ 0 , then $\left(v_{n}\right)_{n}$ is a Cauchy sequence in $H_{\#}^{1}(\mathbb{R})$ and therefore there is $v \in H_{\#}^{1}(\mathbb{R})$ such that $v=\lim _{n \rightarrow+\infty} v_{n}$ in $H_{\#}^{1}(\mathbb{R})$. It remains to prove that $v$ belongs to $X_{\alpha}$. This follows easily by letting $n \rightarrow+\infty$ in the equality $\alpha \int_{P} v_{n}(x) \mathrm{d} x+\int_{P} g\left(x, v_{n}(x)\right) \mathrm{d} x+\int_{P} f(x) \mathrm{d} x=0$ since $g$ is Lipschitz w.r.t. the second variable. We prove now that $T_{\alpha}$ is a contraction if $\alpha$ is small enough. Consider $v_{1}, v_{2} \in X_{\alpha}$ and $u_{1}=T_{\alpha} v_{1}, u_{2}=T_{\alpha} v_{2}$. We have

$$
\begin{aligned}
\alpha^{2}\left(u_{1}(x)-u_{2}(x)\right) & -\left(u_{1}^{\prime \prime}(x)-u_{2}^{\prime \prime}(x)\right)+\alpha\left(g\left(x, u_{1}(x)\right)-g\left(x, u_{2}(x)\right)\right) \\
& =\alpha(1+\alpha)\left(v_{1}(x)-v_{2}(x)\right)+(1+\alpha)\left(g\left(x, v_{1}(x)\right)-g\left(x, v_{2}(x)\right)\right)
\end{aligned}
$$


which implies

$$
\int_{P}\left|u_{1}^{\prime}(x)-u_{2}^{\prime}(x)\right|^{2} \mathrm{~d} x \leq(\alpha+K)(1+\alpha) \int_{P}\left|v_{1}(x)-v_{2}(x)\right|\left|u_{1}(x)-u_{2}(x)\right| \mathrm{d} x .
$$

Using (45) yields $d^{2}\left(u_{1}, u_{2}\right) \leq(\alpha+K)(1+\alpha) \frac{L_{1}^{2}}{2} d\left(u_{1}, u_{2}\right) d\left(v_{1}, v_{2}\right)$ and thus

$$
d\left(T_{\alpha} v_{1}, T_{\alpha} v_{2}\right) \leq(1+\alpha)(\alpha+K) \frac{L_{1}^{2}}{2} d\left(v_{1}, v_{2}\right), \quad \forall v_{1}, v_{2} \in X_{\alpha} .
$$

Therefore if $\alpha$ is small enough such that $(1+\alpha)(\alpha+K) \frac{L_{1}^{2}}{2}<1$ there is a unique fixed point $u_{\alpha} \in X_{\alpha}$ for $T_{\alpha}$, meaning that

$$
-\alpha u_{\alpha}(x)-u_{\alpha}^{\prime \prime}(x)-g\left(x, u_{\alpha}(x)\right)=f(x), \quad x \in \mathbb{R} .
$$

We are looking now for uniform estimates of $\left(u_{\alpha}\right)_{\alpha}$. We can write

$$
-\alpha\left(u_{\alpha}(x)-v_{\alpha}\right)-u_{\alpha}{ }^{\prime \prime}(x)=g\left(x, u_{\alpha}(x)\right)-g\left(x, v_{\alpha}\right)+\alpha v_{\alpha}+g\left(x, v_{\alpha}\right)+f(x), \quad x \in \mathbb{R},
$$

and therefore

$$
\begin{aligned}
\int_{P}\left|u_{\alpha}{ }^{\prime}(x)\right|^{2} \mathrm{~d} x & \leq(\alpha+K) \int_{P}\left|u_{\alpha}(x)-v_{\alpha}\right|^{2} \mathrm{~d} x \\
& +\int_{P}\left(\alpha v_{\alpha}+g\left(x, v_{\alpha}\right)+f(x)\right)\left(u_{\alpha}(x)-v_{\alpha}\right) \mathrm{d} x .
\end{aligned}
$$

As in the proof of Proposition 2.5 (see (33)) since $\int_{P}\left\{\alpha v_{\alpha}+g\left(x, v_{\alpha}\right)+f(x)\right\} \mathrm{d} x=0$ we have

$$
\int_{P}\left\{\alpha v_{\alpha}+g\left(x, v_{\alpha}\right)+f(x)\right\}\left\{u_{\alpha}(x)-v_{\alpha}\right\} \mathrm{d} x \leq\left\|u_{\alpha}^{\prime}\right\|_{L_{\#}^{2}(\mathbb{R})} L_{1}\left\|\alpha v_{\alpha}+g\left(\cdot, v_{\alpha}\right)+f\right\|_{L_{\#}^{2}(\mathbb{R})} .
$$

Combining (45), (47) and (48) yields

$$
\left\|u_{\alpha}{ }^{\prime}\right\|_{L_{\#}^{2}(\mathbb{R})}^{2} \leq(\alpha+K) \frac{L_{1}^{2}}{2}\left\|u_{\alpha}^{\prime}\right\|_{L_{\#}^{2}(\mathbb{R})}^{2}+L_{1}\left\|\alpha v_{\alpha}+g\left(\cdot, v_{\alpha}\right)+f\right\|_{L_{\#}^{2}(\mathbb{R})}\left\|u_{\alpha}{ }^{\prime}\right\|_{L_{\#}^{2}(\mathbb{R})} .
$$

By taking into account that $K L_{1}^{2}<2$ we obtain for $\alpha>0$ small enough

$$
\left\|u_{\alpha}^{\prime}\right\|_{L_{\#}^{2}(\mathbb{R})} \leq \frac{L_{1}}{1-\frac{L_{1}^{2}(\alpha+K)}{2}}\left\|\alpha v_{\alpha}+g\left(\cdot, v_{\alpha}\right)+f\right\|_{L_{\#}^{2}(\mathbb{R})} .
$$

By (45) we deduce also that

$$
\left\|u_{\alpha}-v_{\alpha}\right\|_{L_{\#}^{2}(\mathbb{R})} \leq \frac{L_{1}}{\sqrt{2}}\left\|u_{\alpha}{ }^{\prime}\right\|_{L_{\#}^{2}(\mathbb{R})}, \quad\left\|u_{\alpha}-v_{\alpha}\right\|_{L^{\infty}(\mathbb{R})} \leq L_{1}^{\frac{1}{2}}\left\|u_{\alpha}^{\prime}\right\|_{L_{\#}^{2}(\mathbb{R})},
$$

and since $u_{\alpha}^{\prime \prime}=-\alpha u_{\alpha}-g\left(\cdot, u_{\alpha}\right)-f$ thus $\left(u_{\alpha}\right)_{\alpha}$ is bounded in $H_{\#}^{2}(\mathbb{R})$. We can extract a sequence $\left(\alpha_{k}\right)_{k}$ converging to 0 such that $\lim _{k \rightarrow+\infty} u_{\alpha_{k}}=u$ strongly in $H_{\#}^{1}(\mathbb{R})$ and one gets easily that $u$ is a periodic solution for $-u^{\prime \prime}(x)-g(x, u(x))=f(x)$ in $\mathbb{R}$. Moreover $u$ verifies the estimates

$$
\left\|u-\tilde{u}_{0}\right\|_{L_{\#}^{2}(\mathbb{R})} \leq \frac{\sqrt{2} L_{1}^{2}}{2-L_{1}^{2} K}\left\|g\left(\cdot, \tilde{u}_{0}\right)+f\right\|_{L_{\#}^{2}(\mathbb{R})},\left\|u-\tilde{u}_{0}\right\|_{L^{\infty}(\mathbb{R})} \leq \frac{2 L_{1}^{\frac{3}{2}}}{2-L_{1}^{2} K}\left\|g\left(\cdot, \tilde{u}_{0}\right)+f\right\|_{L_{\#}^{2}(\mathbb{R})},
$$




$$
\left\|u^{\prime}\right\|_{L_{\#}^{2}(\mathbb{R})} \leq \frac{2 L_{1}}{2-L_{1}^{2} K}\left\|g\left(\cdot, \tilde{u}_{0}\right)+f\right\|_{L_{\#}^{2}(\mathbb{R})} .
$$

Suppose now that $g$ is strictly increasing w.r.t. $u$ a.e. $x \in \mathbb{R}$ and consider two periodic solutions $u_{1}, u_{2}$. By observing that $\int_{P} g\left(x, u_{1}(x)\right) \mathrm{d} x=\int_{P} g\left(x, u_{2}(x)\right) \mathrm{d} x=-\int_{P} f(x) \mathrm{d} x$ we deduce that

$$
u_{1}\left(y_{0}\right)=u_{2}\left(y_{0}\right)
$$

for some $y_{0} \in P$. As in (45) we have

$$
\int_{P}\left|u_{1}(x)-u_{2}(x)\right|^{2} \mathrm{~d} x \leq \frac{L_{1}^{2}}{2} \int_{P}\left|u_{1}^{\prime}(x)-u_{2}^{\prime}(x)\right|^{2} \mathrm{~d} x,
$$

and by using the equations of $u_{1}, u_{2}$ we deduce easily that

$$
\int_{P}\left|u_{1}^{\prime}(x)-u_{2}^{\prime}(x)\right|^{2} \mathrm{~d} x \leq \frac{L_{1}^{2} K}{2} \int_{P}\left|u_{1}^{\prime}(x)-u_{2}^{\prime}(x)\right|^{2} \mathrm{~d} x,
$$

which implies that $u_{1}^{\prime}=u_{2}^{\prime}$. The uniqueness follows from (49).

\section{Numerical Simulations}

In this section we compute numerically periodic solutions for $-u^{\prime \prime}(x)+g(x, u(x))=0, x \in \mathbb{R}$, where $g$ is monotone w.r.t. $u$.

\subsection{Numerical approximations for nondecreasing nonlinearities}

The idea is to solve for large enough time the nonlinear parabolic equation

$$
\left.\alpha u+\partial_{t} u-\partial_{x}^{2} u+g(x, u(x, t))=0, \quad(x, t) \in \mathbb{R} \times\right] 0,+\infty[
$$

with an arbitrary periodic initial condition

$$
u(x, 0)=u_{0}(x), \quad x \in \mathbb{R},
$$

and $\alpha>0$. Indeed, if $U$ is the periodic solution of

$$
\alpha U(x)-U^{\prime \prime}(x)+g(x, U(x))=0, \quad x \in \mathbb{R},
$$

we deduce that

$$
\left.\alpha(u-U)+\partial_{t}(u-U)-\partial_{x}^{2}(u-U)+g(x, u(x, t))-g(x, U(x))=0, \quad(x, t) \in \mathbb{R} \times\right] 0,+\infty[,
$$

and after multiplication by $u-U$ and integration over one period one gets

$$
\alpha \int_{0}^{L_{1}}|u(x, t)-U(x)|^{2} \mathrm{~d} x+\frac{1}{2} \frac{\mathrm{d}}{\mathrm{d} t} \int_{0}^{L_{1}}|u(x, t)-U(x)|^{2} \mathrm{~d} x+\int_{0}^{L_{1}}\left|\partial_{x} u-U^{\prime}\right|^{2} \mathrm{~d} x \leq 0, t>0 .
$$

We deduce that $\|u(\cdot, t)-U\|_{L_{\#}^{2}(\mathbb{R})} \leq \mathrm{e}^{-\alpha t}\left\|u_{0}-U\right\|_{L_{\#}^{2}(\mathbb{R})}, t>0$, and thus in order to get a good approximation for $U$ we need to solve (50) for $t_{\alpha} \approx \frac{1}{\alpha} \rightarrow+\infty$ as $\alpha \searrow 0$. A better approach is to replace the constant $\alpha$ by a nonnegative, nonincreasing function $\alpha:\left[0,+\infty\left[\rightarrow \mathbb{R}\right.\right.$ satisfying $\lim _{t \rightarrow+\infty} \alpha(t)=0$. The evolution of $\alpha(\cdot)$ can be 
given by a equation of type

$$
\alpha^{\prime}(t)+\alpha(t) C(u(\cdot, t))=0, \quad t>0,
$$

where $C$ is a nonnegative function verifying $C \approx 1$ if $u(\cdot, t)$ is "almost" periodic w.r.t. $x$ and $C \approx 0$ otherwise. The point is to keep $\alpha$ constant until $u(\cdot, t)$ becomes a good approximation of a periodic function after that diminishing $\alpha$ is allowed. A very simple function $C$ satisfying the property mentioned above could be

$$
C(u(\cdot, t))=\mathbf{1}_{\{|\alpha(t)\langle u(\cdot, t)\rangle+\langle g(\cdot, u(\cdot, t))\rangle|<\varepsilon\}},
$$

with $\varepsilon>0$ a small parameter. Indeed, if $u$ is near a stationary periodic w.r.t. $x$ function, then $\partial_{t} u \approx 0$, $\int_{0}^{L_{1}} \partial_{x}^{2} u \mathrm{~d} x \approx 0$ and by taking the average over one period of $(50)$ one gets that $\alpha(t)\langle u(\cdot, t)\rangle+\langle g(\cdot, u(\cdot, t))\rangle \approx 0$ which implies that $\mathbf{1}_{\{|\alpha(t)\langle u(\cdot, t)\rangle+\langle g(\cdot, u(\cdot, t))\rangle|<\varepsilon\}}=1$. We obtain the following equation for $\alpha(\cdot)$

$$
\alpha^{\prime}(t)+\alpha(t) \mathbf{1}_{\{|\alpha(t)\langle u(\cdot, t)\rangle+\langle g(\cdot, u(\cdot, t))\rangle|<\varepsilon\}}=0, \quad t>0,
$$

supplemented by an initial condition

$$
\alpha(0)=\alpha_{0}>0 .
$$

Therefore our method consists in solving the system (50), (52), with the initial conditions (51), (53). Let us analyze the following two examples

$$
\begin{gathered}
u_{1}(x)=\sin x-\cos x, u_{2}(x)=\sin x+\cos x, \quad x \in \mathbb{R}, \\
g_{1}(x, u)=u(1+\cos x)-(2+\cos x)(\sin x-\cos x), \quad(x, u) \in \mathbb{R}^{2}, \\
g_{2}(x, u)=\frac{u}{\sqrt{1+u^{2}}}(1+\cos x)-(\sin x+\cos x)\left(1+\frac{1+\cos x}{\sqrt{2+\sin (2 x)}}\right), \quad(x, u) \in \mathbb{R}^{2} .
\end{gathered}
$$

We check easily that $\left(u_{k}\right)_{1 \leq k \leq 2}$ are exact $2 \pi$ periodic solutions for $-u_{k}^{\prime \prime}(x)+g_{k}\left(x, u_{k}(x)\right)=0, x \in \mathbb{R}, 1 \leq k \leq 2$. We use the finite differences method i.e.,

$$
\partial_{t} u \approx \frac{u_{i}^{n+1}-u_{i}^{n}}{\Delta t}, \partial_{x}^{2} u \approx \frac{u_{i+1}^{n}-2 u_{i}^{n}+u_{i-1}^{n}}{(\Delta x)^{2}}
$$

where $u_{i}^{n}=u\left(x_{i}, t_{n}\right), x_{i}=i \Delta x, t_{n}=n \Delta t, \forall i \in \mathbb{Z}, \forall n \in \mathbb{N}$. In both cases we start from $u_{0}(x)=1000+$ $\sin x-\sqrt{1+\cos x}, \alpha_{0}=1, x \in \mathbb{R}$, we take $N_{x}=25$ mesh points over one period, $\Delta x=2 \pi / N_{x}$, a time step $\Delta t=0.45(\Delta x)^{2}$. The numerical results after $N_{t}=500$ time steps in the first and second case are illustrated in the Figures 1 and 2 respectively.

\subsection{Numerical approximations for nonincreasing nonlinearities}

In this section we compute numerically the periodic solutions of the envelope equation in one dimension

$$
-u^{\prime \prime}(x)-g(x, u(x))=0, \quad x \in \mathbb{R},
$$

where $g(x, u)=a(1+\cos x) u-\frac{1}{u}-\frac{b}{u^{3}}$, with $a>0, b \geq 0$. We are looking for periodic positive solutions for (54). Actually we will construct solutions satisfying $u(x) \geq r, x \in \mathbb{R}$ for some $r>0$. In order to apply the theoretical results of Section 3 we define the function $g_{r}: \mathbb{R} \times \mathbb{R} \rightarrow \mathbb{R}$ given by $g_{r}(x, u)=g(x, u) \mathbf{1}_{\{u \geq r\}}+g(x, r) \mathbf{1}_{\{u<r\}}$, $(x, u) \in \mathbb{R} \times \mathbb{R}$. Note that $g_{r}$ is continuous and nondecreasing w.r.t. $u$ for any $x \in \mathbb{R}, 2 \pi$ periodic w.r.t. $x$ and Lipschitz w.r.t. $u$ of constant $K=2 a+\frac{1}{r^{2}}+\frac{3 b}{r^{4}}$. We study the modified envelope equation

$$
-u^{\prime \prime}(x)-g_{r}(x, u(x))=0, \quad x \in \mathbb{R} .
$$



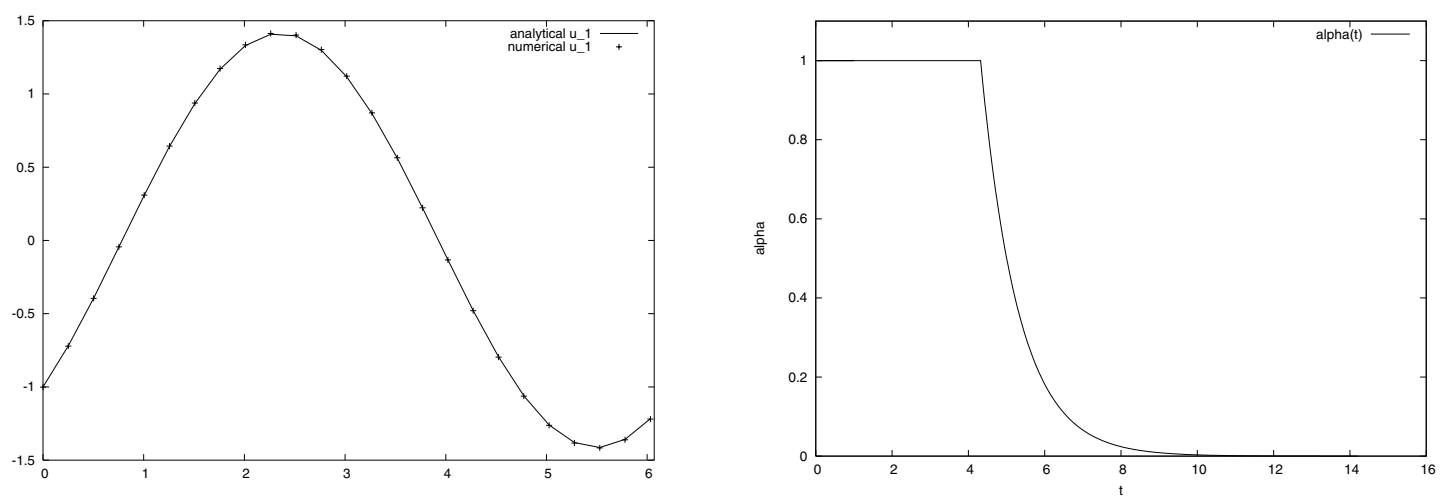

FiguRE 1. Exact/numerical solution $u_{1}$ and time evolution of parameter $\alpha_{1}$.
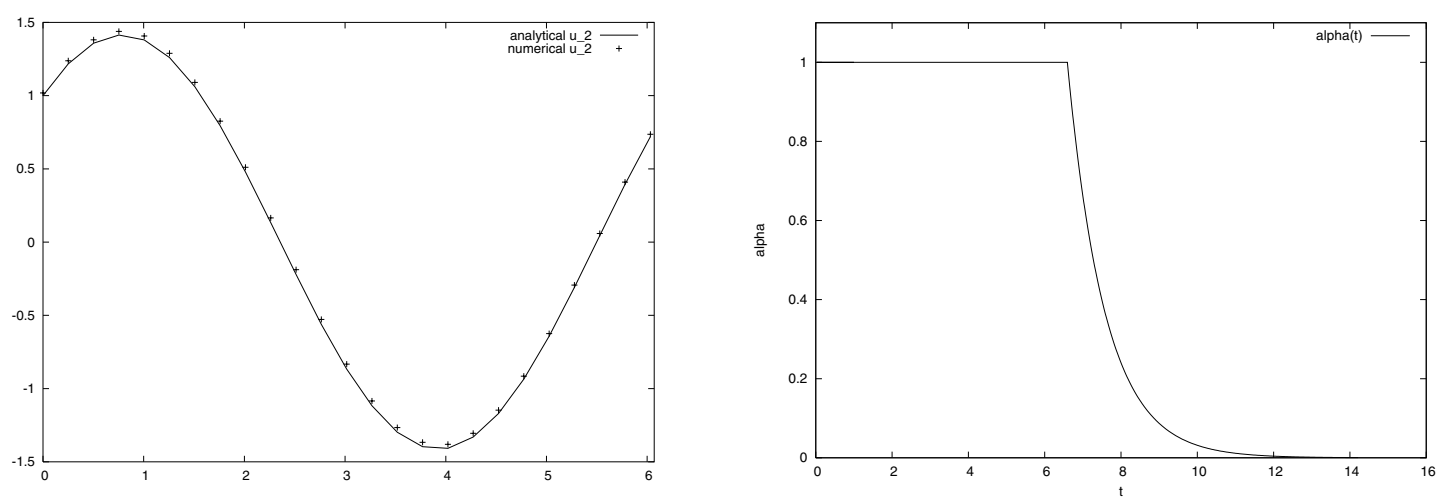

FiguRE 2. Exact/numerical solution $u_{2}$ and time evolution of parameter $\alpha_{2}$.

Observe that the condition $\left(C_{2}^{\prime}\right)$ is satisfied i.e., $\exists u_{0} \in \mathbb{R}$ such that $\int_{0}^{2 \pi} g_{r}\left(x, u_{0}\right) \mathrm{d} x=0$ for $u_{0}=$ $\sqrt{1+\sqrt{1+4 a b}} / \sqrt{2 a}$ provided that

$$
\frac{\sqrt{1+\sqrt{1+4 a b}}}{\sqrt{2 a}}>r .
$$

We intend to apply Theorem 3.1 and therefore we need to impose the condition

$$
1-\frac{(2 \pi)^{2} K}{2}=1-2 \pi^{2}\left(2 a+\frac{1}{r^{2}}+\frac{3 b}{r^{4}}\right)>0
$$

By the above theorem we will deduce the existence of a $2 \pi$ periodic solution satisfying $\left\|u-u_{0}\right\|_{L^{\infty}} \leq$ $\frac{(2 \pi)^{\frac{3}{2}}}{1-2 \pi^{2} K}\left\|g_{r}\left(\cdot, u_{0}\right)\right\|_{L_{\#}^{2}(\mathbb{R})}$, which implies that

$$
u(x) \geq u_{0}-\frac{(2 \pi)^{\frac{3}{2}}}{1-2 \pi^{2} K}\left\|g_{r}\left(\cdot, u_{0}\right)\right\|_{L_{\#}^{2}(\mathbb{R})} .
$$


Observe that the solution $u$ will satisfy also (54) provided that

$$
\frac{(2 \pi)^{\frac{3}{2}}}{1-2 \pi^{2} K}\left\|g_{r}\left(\cdot, u_{0}\right)\right\|_{L_{\#}^{2}(\mathbb{R})}<u_{0}-r .
$$

Since $g_{r}\left(x, u_{0}\right)=a u_{0} \cos x$ we have $\left\|g_{r}\left(\cdot, u_{0}\right)\right\|_{L_{\#}^{2}(\mathbb{R})}=a u_{0} \sqrt{\pi}$ and therefore we need to impose the condition

$$
a u_{0} 2 \sqrt{2} \pi^{2}<\left(u_{0}-r\right)\left(1-2 \pi^{2} K\right) .
$$

We check that $a=10^{-4}, b=10^{4}, r=10^{2}$ satisfy the conditions (56), (57) and (58).

For the numerical resolution of (55) it is convenient to solve for $t$ large enough the parabolic equation

$$
\left.\partial_{t} u_{\alpha}-\partial_{x}^{2} u_{\alpha}=\alpha u_{\alpha}+g_{r}\left(x, u_{\alpha}(x)\right), \quad(t, x) \in\right] 0,+\infty[\times \mathbb{R},
$$

with the initial condition

$$
u_{\alpha}(0, x)=v_{\alpha}, \quad x \in \mathbb{R}
$$

where $\left(v_{\alpha}\right)_{\alpha}$ is given by $\int_{P}\left\{\alpha v_{\alpha}+g_{r}\left(x, v_{\alpha}\right)\right\} \mathrm{d} x=0$. Indeed, this can be justified at least in the linear case i.e., $g(x, u)=K u+f(x), 0 \leq L_{1}^{2} K<2$. If for $\alpha>0$ small enough we denote by $U_{\alpha}$ the unique periodic solution of

$$
-U_{\alpha}^{\prime \prime}(x)=\alpha U_{\alpha}(x)+K U_{\alpha}(x)+f(x), \quad x \in \mathbb{R},
$$

we have

$$
\partial_{t}\left(u_{\alpha}-U_{\alpha}\right)-\partial_{x}^{2}\left(u_{\alpha}-U_{\alpha}\right)=\alpha\left(u_{\alpha}-U_{\alpha}\right)+K\left(u_{\alpha}-U_{\alpha}\right)
$$

Therefore $\frac{\mathrm{d}}{\mathrm{d} t} \int_{P}\left\{u_{\alpha}(t, x)-U_{\alpha}(x)\right\} \mathrm{d} x=(\alpha+K) \int_{P}\left\{u_{\alpha}(t, x)-U_{\alpha}(x)\right\} \mathrm{d} x$, which implies that

$$
\int_{P}\left\{u_{\alpha}(t, x)-U_{\alpha}(x)\right\} \mathrm{d} x=\exp ((\alpha+K) t) \int_{P}\left\{v_{\alpha}-U_{\alpha}(x)\right\} \mathrm{d} x .
$$

But $\int_{P}(\alpha+K) v_{\alpha} \mathrm{d} x=-\int_{P} f(x) \mathrm{d} x=\int_{P}(\alpha+K) U_{\alpha}(x) \mathrm{d} x$ and we deduce that $\int_{P}\left\{v_{\alpha}-U_{\alpha}(x)\right\} \mathrm{d} x=0$. Finally one gets from (62) that $\int_{P}\left\{u_{\alpha}(t, x)-U_{\alpha}(x)\right\} \mathrm{d} x=0, t>0$ and therefore as in (45) we obtain

$$
\left\|u_{\alpha}(t)-U_{\alpha}\right\|_{L_{\#}^{2}(\mathbb{R})} \leq \frac{L_{1}}{\sqrt{2}}\left\|\partial_{x} u_{\alpha}(t)-U_{\alpha}^{\prime}\right\|_{L_{\#}^{2}(\mathbb{R})}, t>0 .
$$

From (61) we deduce now that for any $t>0$ we have

$$
\frac{1}{2} \frac{\mathrm{d}}{\mathrm{d} t} \int_{P}\left|u_{\alpha}(t, x)-U_{\alpha}(x)\right|^{2} \mathrm{~d} x+\int_{P}\left|\partial_{x} u_{\alpha}(t, x)-U_{\alpha}{ }^{\prime}(x)\right|^{2} \mathrm{~d} x=\int_{P}(\alpha+K)\left|u_{\alpha}(t, x)-U_{\alpha}(x)\right|^{2} \mathrm{~d} x,
$$

and by using (63) we obtain

$$
\frac{1}{2} \frac{\mathrm{d}}{\mathrm{d} t} \int_{P}\left|u_{\alpha}(t, x)-U_{\alpha}(x)\right|^{2} \mathrm{~d} x+\left(\frac{2}{L_{1}^{2}}-\alpha-K\right) \int_{P}\left|u_{\alpha}(t, x)-U_{\alpha}(x)\right|^{2} \mathrm{~d} x \leq 0 .
$$

Finally one gets

$$
\left\|u_{\alpha}(t)-U_{\alpha}\right\|_{L_{\#}^{2}(\mathbb{R})} \leq\left\|v_{\alpha}-U_{\alpha}\right\|_{L_{\#}^{2}(\mathbb{R})} \exp \left(-t \frac{2-(\alpha+K) L_{1}^{2}}{L_{1}^{2}}\right), t>0,
$$

and therefore for any $\alpha>0$ small enough we have $\lim _{t \rightarrow+\infty}\left\|u_{\alpha}(t)-U_{\alpha}\right\|_{L_{\#}^{2}(\mathbb{R})}=0$. As before we can replace the constant $\alpha$ by a function $\alpha(\cdot)$ depending on $t$ and therefore we obtain the system (59), (52) with the initial 


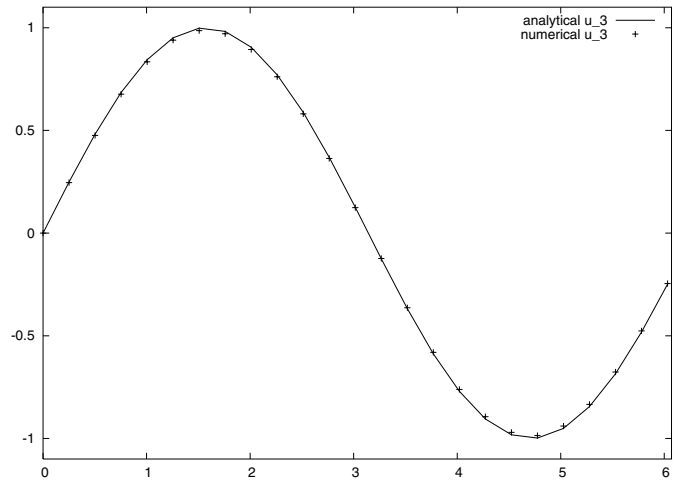

FigURE 3. Exact/numerical solution $u_{3}$.

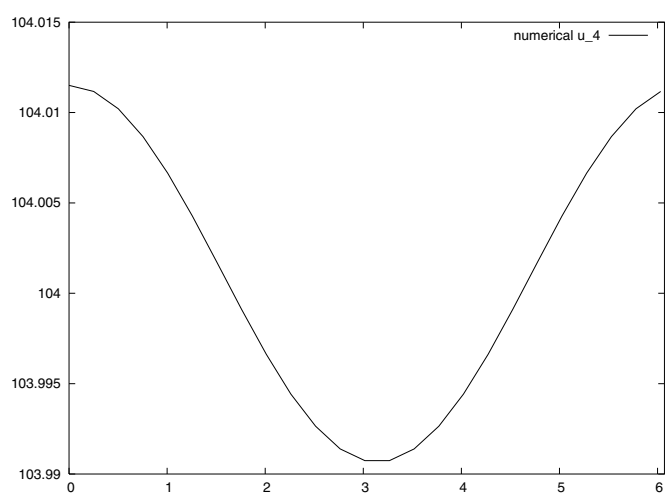

Figure 4: Numerical solution $u_{4}$.

conditions (60) and (53).

Before computing the periodic solutions of the envelope equation let us analyze the periodic solutions for the nonlinearity

$$
g_{3}(x, u)=-K \frac{u}{\sqrt{1+u^{2}}}-\sin x+K \frac{\sin x}{\sqrt{1+\sin ^{2} x}}, \quad(x, u) \in \mathbb{R}^{2} .
$$

Observe that $u_{3}(x)=\sin x$ solves $-u_{3}^{\prime \prime}(x)+g_{3}\left(x, u_{3}(x)\right)=0, x \in \mathbb{R}$. We start from $u_{0}(x)=0, x \in \mathbb{R}, \alpha_{0}=1$, we take $N_{x}=25$ mesh points over one period, $\Delta x=2 \pi / N_{x}, \Delta t=0.45(\Delta x)^{2}$. The results obtained after $N_{t}=200$ time steps are shown in Figure 3 .

Let us discuss now the envelope equation. Notice that for any $\alpha>0$ we have

$$
v_{\alpha}=\frac{\sqrt{1+\sqrt{1+4(a+\alpha) b}}}{\sqrt{2(a+\alpha)}} .
$$

We take $a=10^{-4}, b=10^{4}, r=10^{2}, \alpha_{0}=10^{-6}, u_{0}(x)=v_{\alpha_{0}} \approx 104>r, x \in \mathbb{R}, N_{x}=25, \Delta x=2 \pi / N_{x}$, $\Delta t=0.45(\Delta x)^{2}$. The numerical results after $N_{t}=200$ time steps are illustrated in Figure 4.

\section{REFERENCES}

[1] M. Bostan, Solutions périodiques des équations d'évolution. C. R. Acad. Sci., Ser. I, Math. 332 (2001) 401-404.

[2] M. Bostan, Periodic solutions for evolution equations. Electron. J. Diff. Eqns., Monograph 3 (2002) 41.

[3] H. Brezis, Problèmes unilatéraux. J. Math. Pures Appl. 51 (1972) 1-64.

[4] R.C. Davidson and H. Qin, Physics of charged particle beams in high energy accelerators. Imperial College Press, World Scientific Singapore (2001).

[5] P. Degond and P.-A. Raviart, On the paraxial approximation of the stationary Vlasov-Maxwell system, Math. Models Meth. Appl. Sci. 3 (1993) 513-562.

[6] F. Filbet and E. Sonnendrücker, Modeling and numerical simulation of space charge dominated beams in the paraxial approximation. Research report INRIA, No. 5547 (2004).

[7] I.M. Kapchinsky and V.V. Vladimirsky, Proceedings of the 9th international conference on high energy accelerators, CERN Geneva (1959) 274.

[8] D. Kinderlehrer and G. Stampacchia, An introduction to variational inequalities and their applications. Academic Press, New York, London (1980).

[9] G. Laval, S. Mas-Gallic and P.-A. Raviart, Paraxial approximation of ultra-relativistic intense beams. Numer. Math. 1 (1994) $33-60$.

[10] J.-L. Lions, Quelques méthodes de résolution des problèmes aux limites non-linéaires. Dunod Gauthier-Villars (1969). 
[11] Z. Meiyue, C. Taiyoung, L. Wenbin and J. Yong, Existence of positive periodic solution for the electron beam focusing system. Math. Meth. Appl. Sci. 28 (2005) 779-788.

[12] A. Nouri, Paraxial approximation of the Vlasov-Maxwell system: laminar beams. Math. Models Meth. Appl. Sci. 4 (1994) 203-221.

[13] P.-A. Raviart, Paraxial approximation of the stationary Vlasov-Maxwell equations, Nonlinear partial differential equations and their applications. Collège de France Seminar, vol. XIII Paris (1991-1993), Pitman Res. Notes Math. Ser., Longman Sci. Tech., Harlow 302 (1994) 158-171.

[14] M. Reiser, Theory and design of charged-particle beams. Wiley, New York (1994). 